\title{
BASICS OF QUANTUM MECHANICS, GEOMETRIZATION AND SOME APPLICATIONS TO QUANTUM INFORMATION
}

\author{
JESÚS CLEMENTE-GALLARDO AND GIUSEPPE MARMO
}

\begin{abstract}
In this paper we present a survey of the use of differential geometric formalisms to describe Quantum Mechanics. We analyze Schrödinger framework from this perspective and provide a description of the Weyl-Wigner construction. Finally, after reviewing the basics of the geometric formulation of quantum mechanics, we apply the methods presented to the most interesting cases of finite dimensional Hilbert spaces: those of two, three and four level systems (one qubit, one qutrit and two qubit systems). As a more practical application, we discuss the advantages that the geometric formulation of quantum mechanics can provide us with in the study of situations as the functional independence of entanglement witnesses.
\end{abstract}

\section{INTRODUCTION}

Interference phenomena of material particles (matter waves) as electrons, neutrons, atoms, etc provide the most striking evidence for the need to elaborate a new mechanics which goes beyond and encompasses classical mechanics.

At the same time, phenomena like photoelectric and Compton effects show a "corpuscular" behavior of radiation requiring, therefore, the need for a revision of the classical description of radiation.

The associated quantitative results suggest that wave-like and corpuscular-like attributes satisfy the following Einstein- de Broglie relation

$$
p_{a} d x^{a}-E d t=\hbar\left(k_{a} d x^{a}-\omega d t\right)
$$

where $\left(x^{a}, p_{a}\right)$ are the coordinates of the phase space and $t$ stands for the time. This relation between the Poincaré one form on the phase-space over the space-time and the optical phase-space establishes a relation between momentum $\left(p_{a}\right)$ and energy $(E)$ of the corpuscular behavior and the wave-number $\left(k_{a}\right)$ and frequency $(\omega)$ of the wave behavior. The dimensional proportionality coefficient is the Planck constant.

We may use this relation to predict under which experimental conditions light will behave like a "corpuscule" and an electron will behave like a "wave".

By means of this relation it is possible to conceive of interference experiments like the one of the double slit. When carried on with electrons, we find some peculiar results for which we do not have a simple interpretation in the classical framework. The actual experiment has been performed in such a way that at each time only one electron is present between the source and the screen. It is found that the electron impinges on the screen at "given points" and leave a spot.

All the spots are alike, there are no "half-spots". After a few hundred electrons have passed we find a picture of spots erratically distributed on the screen, as we can see in the following picture 


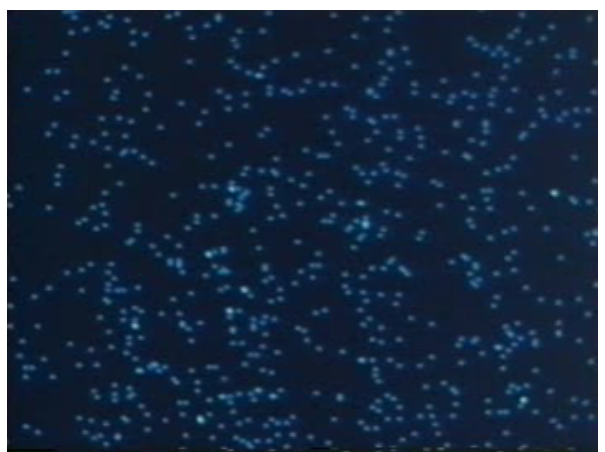

However, with several thousands electrons, we get a very clear interference pattern.

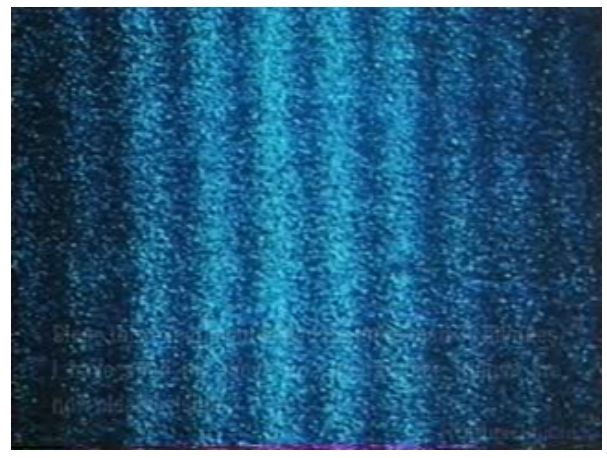

Experiments of this kind have been performed (see [37, 14, 28, 39]).

The same findings occur if we experiment with photons (light quanta), again with an experimental arrangement that makes sure that only one photon is present between source and screen at each time.

These interference aspects call for a theory with a wave-like description, having a statistical-probabilistic character, along with an intrinsically discrete aspect (i.e no half-spots should be possible).

We could try now to perform an experiment to watch which path the electrons follow on its way from the source to the screen. An experimental setup uses a conducting plate and the mirror image of the charge to find out which region will be "heated" by the passage of the electron (see [36]). The setup is as follows:

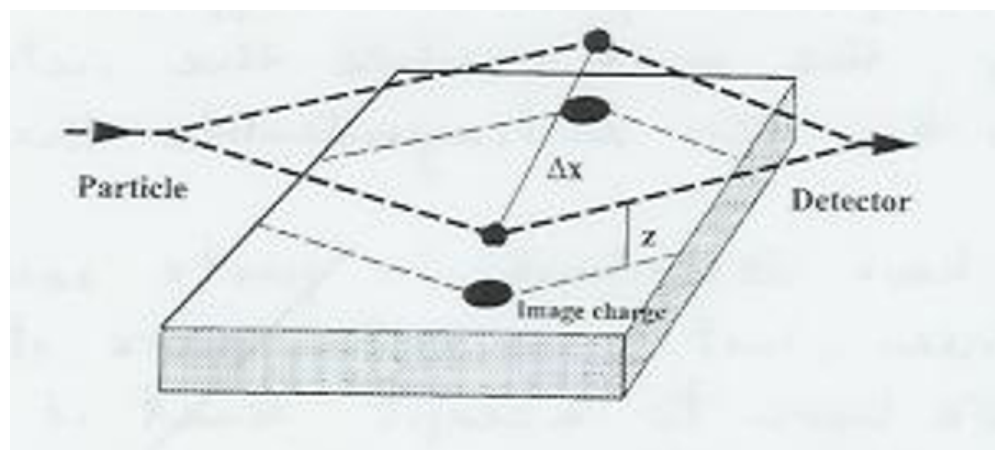

We find that for $z \approx 0$ the interference pattern disappears: 


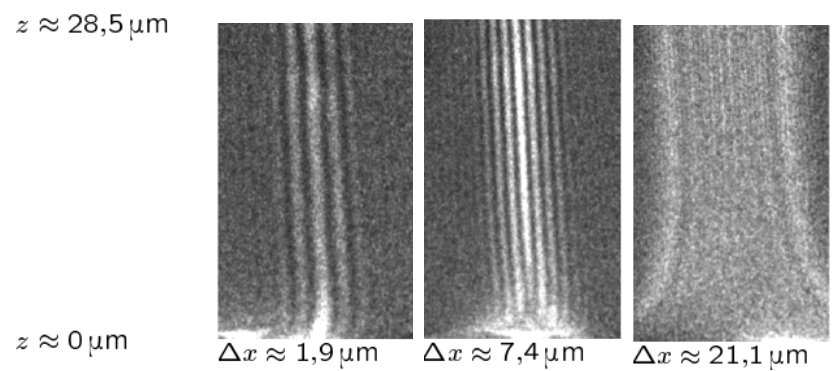

We may try to summarize the main findings of our experiments:

- electrons arrive at the screen in identical lumps

- there is a wave associated with the electrons to describe the interference pattern

- there is a probability for the electron to impinge on the screen within a preassigned region

- under some appropriate experimental conditions we recover a classical-like behavior of the electrons, i.e. the interference pattern disappears. More specifically, the more visible the interference pattern is, the less distinguishable the paths are.

What we have described is quite counter-intuitive for particles, but it is even more startling for light. We have to accept that it is not so simple to elaborate a model capable of accounting for these results within the classical framework. Indeed light provides the most relevant example of the dual nature of quantum objects: its oscillatory properties were suitable to verify the electromagnetic theory of Maxwell, while its lumpiness (the photons) signalled the birth of modern quantum theory. The dualism between the two pictures may appear to be in contradiction, however it constitutes the first example of what is known as complementarity in quantum theory, i.e. the possibility to display both wave and corpuscular properties.

From the historical point of view, things went differently because inconsistencies arose already in the derivation of the spectral distribution of energy density of a black body radiation. Max Planck proposed his well known ad-hoc modification of the standard phenomenological approach. His result, however, did not explain the physics behind the successful result.

Another deficiency of the classical theory arose at the level of the models available for atoms and molecules, where it was not possible to account for the stability of atoms and molecules while explaining the detected atomic spectra. An account of the experimental background material can be found in most textbooks of Quantum Mechanics, for instance in ([17]).

Various efforts of the youngest theoretical physicists of that period gave rise to two alternative, but equivalent, formulations of quantum mechanics, usually associated to the names of Schrödinger and Heisenberg. As it is usually the case, though, the final theory grew out of the efforts of a reasonable large community of theoretical physicists during the first half of the XXth century.

This paper is an expanded version of some lectures delivered by one of us (G. M.) at the "Advanced Winter School on the Mathematical Foundation of Quantum Control and Quantum Information" which took place at Castro Urdiales (Spain), February 11-15, 2008. The audience was composed of PhD students in Mathematics 
and in Physics, and this explains the pedagogical nature of this paper. The content of the lectures has been revised and expanded in a substantial way by both of us.

\section{SCHRÖDINGER'S WAVE MECHANICS}

2.1. Introduction. Of course, if there are waves to describe the wave-like behavior, there ought to be a wave equation to describe the evolution of the system in time. This equation is called the Schrödinger equation and has the following structure:

$$
\frac{d}{d t} \psi=-\frac{i}{\hbar} H \psi
$$

The solutions, as functions of time, describe the time evolution of the initial conditions of the system. The complex valued function $\psi$ is called the wave function and is defined on the configuration space of the system we are considering. From a physical point of view it is interpreted as a probability amplitude, but this interpretation requires, for consistency, that

$$
\int_{D} \psi^{*} \psi d \mu=1,
$$

i.e. the probabilistic interpretation requires $\psi^{*} \psi d \mu$ to be a probability density and $\psi$ to be square integrable on the domain $D$ where our physical system is defined. We conclude thus that wave functions representing physical systems must be elements of the Hilbert space of square integrable functions defined on the configuration space $D$. We shall denote this space as $\mathcal{H}=\mathcal{L}^{2}(D)$. The operator $H$ is usually called the Hamiltonian operator and it acts on wave functions as a linear differential operator. The spectrum will be real, if $H$ is self-adjoint. If it is unbounded and if its domain of definition does not coincide with $\mathcal{H}$ we say that we have to "face domain problems". The requirement of linearity arises from the superposition rule usually associated with the description of interference phenomena.

To avoid dealing with unbounded operators, often one starts from the "exponentiated version" of our evolution equation, i.e. a formal solution of the equation 2 , which we write as

$$
\psi(t)=U\left(t, t_{0}\right) \psi\left(t_{0}\right) .
$$

If $H$ does not depend on time the expression of the evolution operator $U\left(t, t_{0}\right)$ may be written as:

$$
U\left(t, t_{0}\right)=e^{-\frac{i}{\hbar} H\left(t-t_{0}\right)}
$$

In this form the evolution operator is bounded and always reversible. It defines a one-parameter group of unitary transformations on the Hilbert space defined by the wave functions. Unitarity of the evolution means that such evolution is compatible with the probabilistic interpretation. Starting with the evolution expressed as a one-parameter group of unitary transformations, the operator $H$, which defines the Schrödinger equation, emerges as the infinitesimal generator of the group associated with the evolution.

Thus we have identified the basic ingredients appearing in the Schrödinger picture of Quantum Mechanics. It is important to remark the presence of the new fundamental constant $\hbar$ in the description of this class of phenomena. It implies some fundamentally new aspects of the quantum theory with respect to the classical theory. For instance it is known that any measurement process requires an exchange of energy (or information) between the object we are measuring and the measurement apparatus. The presence of $\hbar$ implies that these exchanges can not be 
made arbitrarily small and therefore idealized to be negligible. Thus, in the measurement process, we can not conceive of a sharp separation between the "object" and the "apparatus", so that we can disregard the apparatus altogether. Moreover, in the measuring process, there is an inherent ambiguity in the "cut" between what we identify as "object" and what we identify as the "apparatus".

The problem of measurement in quantum theory is a very deep one and goes beyond the scope of these notes. We may simple mention that within the von Neumann formulation of Quantum Mechanics the "measurement" gives rise to the so called "collapse of the wave function". When we measure some dynamical variable (i.e. a physical quantity), the wave function which represents the system is projected onto one of the eigenstates of the operator $A$ with a probability that can be computed. However we shall not insist on these problem any longer and move to the general mathematical structures emerging in the description of quantum mechanical systems.

While we shall deal mainly with finite dimensional Hilbert spaces, we are going to consider very briefly the Weyl-Wigner formalism in infinite dimensions and mention the tomographic formulation of Quantum Mechanics. The reason is that this formulation is the most suitable for the study of the quantum-classical transition.

Before closing this introduction and to better put into perspective the Schrödinger and the Heisenberg pictures, we are going to make a few general considerations on the minimal mathematical structures required for the description of a physical system. From a minimalist point of view, we identify three main ingredients:

- a space of states, which we define as $\mathcal{S}$,

- a space of observables, which we denote as $\mathcal{O}$,

- a real valued pairing $\mu: \mathcal{O} \times \mathcal{S} \rightarrow \mathbb{R}$. This pairing produces a real number out of a state and an observable, and it is associated with the measurement process. To be more precise, $\mu(A, \psi)$, for $A \in \mathcal{O}$ and $\psi \in \mathcal{S}$, will be a probability measure on the Borel subsets of $\mathbb{R}$.

It is not difficult to identify these objects in the framework of classical dynamics or of classical statistical mechanics. In Quantum Mechanics we have two prevailing pictures:

- The Schrödinger picture. In this case $\mathcal{S}$ is identified with the vectors of a Hilbert space $\mathcal{H}$, while the set of dynamical variables (the observables) is a derived concept: they are identified with self-adjoint operators acting on $\mathcal{H}$.

- The Heisenberg picture. Here the situation is complementary: the set of dynamical variables (the observables) is the primary concept. Observables are assumed to be the real elements of a $\mathbb{C}^{*}$-algebra $\mathcal{A}$. The states are then a derived concept and are defined as a proper subset of the space of linear functionals on $\mathcal{A}$.

2.2. The mathematical structure of the Schrödinger picture. In this picture the relevant carrier space is a Hilbert space $\mathcal{H}$. Very often, it is realized as a space of square-integrable complex valued functions defined on some spatial domain $D \subset \mathbb{R}^{n}$, identified with the configuration space of our system. This configuration space turns out to be the configuration space of the physical system that we would identify in the quantum-classical transition when considering the so-called classical limit defined as $\hbar \rightarrow 0$. 
States are unitary vectors, which are identified when they differ by multiplication by a complex number of modulus one (i.e. an overall phase). This implies an equivalence relation on $\mathcal{H}$ : given $\psi_{1}$ and $\psi_{2}$ in $\mathcal{H}$ satisfying $\left\langle\psi_{1} \mid \psi_{1}\right\rangle=1=\left\langle\psi_{2} \mid \psi_{2}\right\rangle$, we have

$$
\psi_{2}=e^{i \theta} \psi_{1} \quad \theta \in[0,2 \pi) \Rightarrow \psi_{1} \sim \psi_{2} .
$$

As a result, physical states are identified with points in the complex projective space associated to $\mathcal{H}$. We shall denote it as $\mathcal{P} \mathcal{H}$ or occasionally $\mathcal{R}(\mathcal{H})$ (meaning the space of rays of $\mathcal{H}$ ). The corresponding equivalence classes will be denoted as

$$
[\psi]=\{\phi \in \mathcal{H} \mid \phi \sim \psi\}
$$

On the other hand, observables are defined as self-adjoint operators acting on $\mathcal{H}$. The pairing between states and observables is defined in terms of the Hermitian structure of $\mathcal{H}$ : with any pair $([\psi], A)$ we associate the expectation value function $e_{A}$ and defined as

$$
e_{A}([\psi])=\frac{\langle\psi, A \psi\rangle}{\langle\psi, \psi\rangle},
$$

it is clear that the right hand side depends only on the equivalence class $[\psi]$. We are using here, as in the rest of the paper, the bra-ket notation of Dirac [13.

Evolution is defined on the Hilbert space $\mathcal{H}$ by means of the Scrödinger equation

$$
i \hbar \frac{d}{d t}|\psi\rangle=H|\psi\rangle
$$

We can elaborate a little on this equation. Consider now the equation for the complex conjugate and transpose of the wave function:

$$
-i \hbar \frac{d}{d t}\langle\psi|=\langle\psi| H
$$

where the reality of $H$ has been used.

Combining both equation, it is immediate to see that, at the level of the Hilbert space:

$$
\frac{d}{d t}\langle\psi, \psi\rangle=-\frac{i}{\hbar}(\langle\psi, H \psi\rangle-\langle\psi H, \psi\rangle)
$$

which appears as a continuity equation.

To be specific we consider the Hilbert space realized in terms of wave functions and the Hamiltonian operator as a differential operator associated with a particle moving in a potential $V(\vec{x})$, we find:

$$
i \hbar \frac{d}{d t} \psi=-\frac{\hbar^{2}}{2 \pi i} \Delta \psi+\hat{V}(\vec{x}) \psi
$$

where $\Delta$ is the Laplacian operator defined on $D$ and $\hat{V}(x)$ is the multiplication operator

$$
\hat{V}(\vec{x}) \psi=V(\vec{x}) \psi \quad \psi \in \mathrm{E}^{2}(D)
$$

As we have mentioned, the presence of the configuration space $D$ in the realization of the Hilbert space $\mathcal{H}$ in terms of square integrable functions on $D$ incorporates some shadows of the classical limit. As a matter of fact, if we introduce the polar form of the wave function as

$$
\psi(\vec{x}, t)=A(\vec{x}, t) e^{\frac{i}{\hbar} W(\vec{x}, t)},
$$


where $A$ and $W$ are real valued functions and $\psi^{*} \psi=A^{2}=\rho$, we decompose the complex linear Schrödinger equation into two nonlinear and coupled differential equations. Here the equation for $W$ contains an additional "quantum potential"

i.e.

$$
U(\vec{x}, t)=-\frac{\hbar^{2}}{2 m} \frac{\Delta A}{A},
$$

$$
\frac{d W}{d t}=-\frac{(\operatorname{grad} W)^{2}}{2 m}+V(\vec{x})+U(\vec{x}, t) .
$$

The other equation takes the form of a continuity equation for the probability distribution $\rho$ :

$$
\frac{d \rho}{d t}+\operatorname{div} \rho \vec{v}=0 \quad \vec{v}=\frac{\operatorname{grad} W}{m} .
$$

The vector field associated with the wave function $\psi$ :

$$
\vec{v}=\frac{d \vec{x}}{d t}=\frac{i \hbar}{m} \frac{\psi^{*} \nabla \psi-\psi \nabla \psi^{*}}{\psi^{*} \psi}
$$

is the extra ingredient of Bohmian mechanics ([3]).

Let us indulge now on the integration of the Schrödinger equation. When the Hamiltonian operator does not depend explicitly on time, i.e. it is time-translation invariant, we may use the separation of variables and consider

$$
\psi(\vec{x}, t)=\phi(\vec{x}) e^{-\frac{i}{\hbar} E t} .
$$

We thus get the Helmholtz equation associated with the Schrödinger equation:

$$
\left(-\frac{\hbar^{2}}{2 m} \Delta+\hat{V}\right) \phi=E \phi
$$

One solves for this equation in terms of an "eigenvalue problem" with preassigned initial conditions (boundary conditions) and finds, for suitable potentials, a set of fundamental solutions which provide also a basis for the Hilbert space, say

$$
\phi_{1}, \phi_{2}, \cdots
$$

with the corresponding eigenvalues

$$
E_{1}, E_{2}, \cdots
$$

A general solution for our quantum problem will thus have the form:

$$
\psi(\vec{x}, t)=\sum_{j} c_{j} \phi_{j}(\vec{x}) e^{-\frac{i}{\hbar} E_{j} t},
$$

with $\psi(\vec{x}, 0)=\sum_{j} c_{j} \phi_{j}(\vec{x})$ allowing to determine $\left\{c_{j}\right\}$ up to an overall phase. For simplicity we have considered a situation where eigenvalues are not degenerate. The merit of the particular basis we have considered relies on the fact that on these vectors $\hat{H}$ acts as a multiplication operator, i.e. as a diagonal matrix. Let us elaborate a little more on this aspect in more general terms. To this aim it is convenient to go back to the abstract Dirac notation in terms of bras and kets. Thus starting with Schrödinger equation

$$
\frac{d}{d t}|\psi\rangle=\frac{H}{i \hbar}|\psi\rangle
$$

Let us consider now an orthonormal basis for the Hilbert space $\left\{\left|e_{i}\right\rangle\right\}_{i=1, \cdots}$ and the corresponding basis for the dual space $\left\{\left\langle e_{i}\right|\right\}_{i=1, \ldots}$, with the property

$$
\left\langle e_{j} \mid e_{i}\right\rangle=\delta_{j k}
$$


In this basis the Hermitian structure is represented by the identity matrix. The orthogonality also allows to write the decomposition of the identity operator

$$
\mathbb{I}=\sum_{j}\left|e_{j}\right\rangle\left\langle e_{j}\right| .
$$

With the help of these bases we can write our initial Schrödinger equation in the matrix form:

$$
\frac{d}{d t} \sum_{j}\left|e_{j}\right\rangle\left\langle e_{j} \mid \psi\right\rangle=\sum_{j k}\left|e_{j}\right\rangle\left\langle e_{j}\left|\frac{H}{i \hbar}\right| e_{k}\right\rangle\left\langle e_{k} \mid \psi\right\rangle .
$$

Now, by using the fact that $\left\{\left|e_{j}\right\rangle\right\}$ are a basis, we get:

$$
\frac{d}{d t} \psi^{j}=\sum_{k}\left\langle e_{j}\left|\frac{H}{i \hbar}\right| e_{k}\right\rangle \psi^{k}
$$

Denoting $h_{j}^{k}=\sum_{k}\left\langle e_{j}\left|\frac{H}{i \hbar}\right| e_{k}\right\rangle$ we can write the Schrödinger equation as

$$
\frac{d}{d t} \psi^{j}=\sum_{k} h_{k}^{j} \psi^{k}
$$

This equation would allow us to use all the results from matrix algebra if the matrices would be finite dimensional. As a matter of fact, this would be the case if for any chosen $j$ the number of indices $k$ coming into $h_{k}^{j}$ were finite. In geometrical terms this would mean that we can decompose the full Hilbert space into the direct sum of invariant finite dimensional Hilbert spaces. In this way the infinite dimensional matrix associated with the operator would decompose into a block diagonal form, each block being a finite dimensional matrix. Thus the eigenvalue problem associated with the Helmholtz equation is nothing but a way to find such an invariant decomposition of the Hilbert space where, in addition, each finite dimensional block is a multiple of the identity, the multiplication factor being the eigenvalue of the corresponding eigenspace. This procedure gives rise to the well known spectral decomposition of the Hamiltonian operator.

Very often, an invariant decomposition of the full Hilbert space will be associated with the decomposition into irreducible representations of a compact group of symmetries for the Hamiltonian operator.

In conclusion, the most convenient basis $\left\{\left|e_{i}\right\rangle\right\}$ depends on the particular problem we are dealing with, and there are no general prescriptions.

Of course, due to the role of the observer, or apparatus, or laboratory, a convenient basis from the point of view of interpretation, is provided by the eigenstates of the particle's position vector operator $\hat{\vec{X}}$ (whose components are the three commuting coordinate operators $\hat{x}_{1}, \hat{x}_{2}, \hat{x}_{3}$, defined as $\left.\hat{x}_{j}|\vec{x}\rangle=x_{j}|\vec{x}\rangle\right)$.

This continuous basis satisfies some generalized orthonormality conditions, namely

$$
\int|\vec{x}\rangle d^{3} x\langle\vec{x}|=\mathbb{I} \quad\left\langle\vec{x} \mid \vec{x}^{\prime}\right\rangle=\delta\left(\vec{x}-\vec{x}^{\prime}\right) .
$$

Decomposing $|\phi\rangle$ in the $|\vec{x}\rangle$ basis yields

$$
|\phi\rangle=\int|\vec{x}\rangle d^{3} x\langle\vec{x} \mid \phi\rangle .
$$

Usually we write $\langle\vec{x} \mid \phi\rangle=\phi(\vec{x})$ and this basis provides us with a clear interpretation of the wave function, i.e. the component of the abstract vector $|\phi\rangle$ in the basis 
of the position operators. Clearly the continuous index $\vec{x}$ replaces previous discrete indices $k, j$ and integration replaces the summation.

The position representation is quite suitable for measurements of the particle's position. In other instances, as for instance the case of massless particles, the momentum representation is more convenient. In this case we use the momentum operator basis $|\vec{p}\rangle$ associated with the momentum operator $\hat{\vec{P}}$ :

$$
\hat{\vec{P}}|\vec{p}\rangle=\vec{p}|\vec{p}\rangle \text {. }
$$

Again a decomposition of the identity can be written with this basis:

$$
\int|\vec{p}\rangle d^{3} p\langle\vec{p}|=\mathbb{I} \quad\left\langle\vec{p} \mid \vec{p}^{\prime}\right\rangle=\delta\left(\vec{p}-\vec{p}^{\prime}\right) .
$$

Each of these states can be decomposed in the position basis:

$$
|\vec{p}\rangle=\int|\vec{x}\rangle d^{3} x\langle\vec{x} \mid \vec{p}\rangle=\frac{1}{(2 \pi \hbar)^{3 / 2}} \int d^{3} x e^{\frac{i}{\hbar} \vec{p}}|\vec{x}\rangle
$$

Thus the components of the momentum eigenstates, in the position representation, are given by plane-waves. Therefore they are not square-integrable functions and can not be considered as elements of $\mathrm{E}^{2}(D)$. In this formalism, to make sense of the eigenstates of the position or of the momentum operators, one usually deals with wave packets. These are usually written as:

$$
\psi(\vec{x}, t)=\frac{1}{(2 \pi \hbar)^{3 / 2}} \int d^{3} p \phi(\vec{p}) e^{\frac{i}{\hbar}(\vec{p} \vec{x}-E t)},
$$

and $E$ is written in terms of the momenta. The particular expression of $E$ in terms of the momenta is usually called the dispersion relation and the integral appearing in the momentum variables should be considered as taken on the submanifold defined by the dispersion relation.

The Fourier transform which appears in going from one set (of maximally commuting) observables to a conjugate set (of maximally commuting) variables plays a very important role because it is a unitary transformation and therefore compatible with the probabilistic interpretation.

Moreover, from the mathematical point of view, we notice that the Fourier transform represents the "harmonic analysis" on the Abelian vector group. Thus it is associated with translations in space-time or translations in the energy-momentum space [40].

It is interesting to write now the evolution operator we mentioned above (4), when we consider the position representation. We find:

$$
\langle\vec{x} \mid \psi(t)\rangle=\int d^{3} x^{\prime}\left\langle\vec{x}\left|U\left(t, t_{0}\right)\right| \vec{x}^{\prime}\right\rangle\left\langle x^{\prime} \mid \psi\left(t_{0}\right)\right\rangle,
$$

i.e.

$$
\psi(\vec{x}, t)=\int d^{3} x^{\prime} G\left(\vec{x}, \vec{x}^{\prime} ; t, t_{0}\right) \psi\left(\vec{x}^{\prime}, t_{0}\right) .
$$

Here we have introduced the standard notation for the Green function, the "propagator" which makes our initial state to evolve. It simply represents the matrix form of the evolution operator in the continuous basis $|\vec{x}\rangle$ (in particular the matrix element $\left.\left\langle\vec{x}\left|U\left(t, t_{0}\right)\right| \vec{x}^{\prime}\right\rangle\right)$. It is simple to see that at the initial time

$$
G\left(\vec{x}, \vec{x}^{\prime} ; 0\right)=\delta\left(\vec{x}-\vec{x}^{\prime}\right) .
$$


Therefore these matrix elements become functions on $Q \times Q$ ( $Q$ being the configuration space). By using $\int d^{3} x|x\rangle\langle x|$ or $\int d^{3} p|p\rangle\langle p|$, we can find analogous matrix elements written as functions of the cotangent bundle $T^{*} Q$, i.e. $G(\vec{p}, \vec{x} ; t)$ or $G(\vec{x}, \vec{p} ; t)$ respectively.

The probabilistic interpretation we have considered in the introduction is now formalized by saying that when the state of our system is described by the wave function $\psi(\vec{x}, t)$, the probability of finding the particle in the region $D \subset \mathbb{R}^{3}$ at time $t$, is given by

$$
\mathcal{P}(D ; t)=\int_{D}|\psi(\vec{x}, t)|^{2} d^{3} x
$$

The operator

$$
\mathcal{P}_{D}=\int_{D}|\vec{x}\rangle d^{3} x\langle\vec{x}|
$$

is a projection operator and may also be described by means of the characteristic function

$$
\chi_{D}(\vec{x})= \begin{cases}1 & \text { if } \vec{x} \in D \\ 0 & \text { if } \vec{x} \notin D\end{cases}
$$

Thus we can also write:

$$
\mathcal{P}_{D}=\int \chi_{D}(\vec{x})|\vec{x}\rangle d^{3} x\langle\vec{x}|
$$

Similar formulae can also be written for the momentum representation or any other realization of the Hilbert space in terms of square integrable functions.

Up to here, we have been using the position operator representation or the momentum-operator representation and mentioned the possibility of using either one. One may wonder if the general aspects could be dealt with without making an a-priori choice of the representation.

As a matter of fact, it is indeed possible and this is what is done in the WeylWigner formalism along with the further elaboration given by the tomographic formalism.

The reduction to a specific representation is due to the requirement of irreducibility of the representation of the canonical commutation relations. In the following sections we shall briefly consider the Weyl-Wigner formalism, the canonical commutation relations and the emergence of these irreducible representations.

\section{WEYL SYSTEMS}

3.1. Definition and main properties. A Weyl system is defined on a symplectic vector space $(V, \omega)$ in the following way. It is a map

$$
W:(V, \omega) \rightarrow U(\mathcal{H}), \quad v \mapsto W(v),
$$

from the symplectic vector space to unitary operators $U(\mathcal{H})$ on some Hilbert space $\mathcal{H}$. We require that this map defines a strongly continuous one-parameter group of transformations $\mathbb{R} \ni t \mapsto W(t v)$ and that it satisfies the property

$$
W\left(v_{1}\right) W\left(v_{2}\right)=e^{\frac{i}{\hbar} \omega\left(v_{1}, v_{2}\right)} W\left(v_{1}+v_{2}\right) \quad \forall v_{1} v_{2} \in V
$$

Strong continuity, by means of Stone's theorem, implies the existence of an (essentially) selfadjoint operator $R(v)$ such that

$$
W(t v)=e^{i t R(v)}
$$


A well-known theorem by von Neumann establishes that for any finite-dimensional symplectic vector space a Weyl system can always be obtained in terms of operators acting on square-integrable functions defined on any Lagrangian subspace $L \subset V$, with the translational invariant Lebesgue measure $\mu_{L}$. We recall that a Lagrangian subspace of a symplectic vector space is a maximal vector subspace $L$ such that $\omega\left(v_{1}, v_{2}\right)=0$ for any pair of vectors $v_{1}, v_{2} \in L$. Maximality implies that any other vector $u \in V$ such that $\omega(v, u)=0$ for any $v \in L$ must belong to $L$.

It is immediately seen that when vectors $v_{1}, v_{2}$ are such that $\omega\left(v_{1}, v_{2}\right)=0$, the operators $W\left(v_{1}\right)$ and $W\left(v_{2}\right)$ commute. Therefore, a Lagrangian subspace arises as the joint spectrum of a maximal commuting set of unitary operators.

By using vectors $|y\rangle$, eigenvectors of the operators associated with vectors of $L$, we can write a decomposition of the identity

$$
\mathbb{I}=\int_{L}|y\rangle \mu_{L}\langle y|
$$

and also

$$
|\psi\rangle=\int_{L}|y\rangle \mu_{L}\langle y \mid \psi\rangle=\int_{L} \psi(y)|y\rangle \mu_{L}
$$

Within this setting, a Weyl system can be constructed in the following way. We consider the Lagrangian subspace $L \subset V$ and its cotangent bundle $T^{*} L \sim L \times L^{*}$, which is endowed with the canonical symplectic form $\omega_{0}$. Now we construct a symplectomorphism

$$
(V, \omega) \longrightarrow\left(T^{*} L, \omega_{0}\right)
$$

Thus we can write the elements of $V$ as pairs $v=(x, \alpha)$ where $x \in L$ and $\alpha \in$ $L^{*}$. With this decomposition, Weyl operators will be defined by their action on $\mathrm{E}^{2}\left(L, \mu_{L}\right)$ as

$$
\begin{gathered}
{[W(x, 0) \psi](y)=\psi(x+y) \quad x, y \in L \quad \psi \in \mathrm{E}^{2}\left(L, \mu_{L}\right)} \\
{[W(0, \alpha) \psi](y)=e^{i \alpha(y)} \psi(y) \quad y \in L, \alpha \in L^{*} \quad \psi \in \mathrm{E}^{2}\left(L, \mu_{L}\right) .}
\end{gathered}
$$

We know from Stone's theorem that there exist infinitesimal generators for these transformations. We may introduce the operators

$$
\hat{P}^{j}=R\left(\mathfrak{l}_{j} ; 0\right) \quad \hat{Q}_{j}=R\left(0 ; \mathfrak{l}_{j}\right),
$$

where we use $\mathfrak{l}_{j}$ to represent the base vectors for $L$ and $L^{*}$, i.e. $\mathfrak{l}_{j}=(0,0, \cdots, 1,0, \cdots, 0)$, 1 being in the $j$-th position.

With these operators, the Weyl map has the form:

$$
W(x, \alpha)=e^{\frac{i}{\hbar}\left(x_{j} \hat{P}^{j}+\alpha^{j} \hat{Q}_{j}\right)} .
$$

Equivalently we could write instead:

$$
W_{1}(x, \alpha)=e^{\frac{i}{\hbar} \alpha^{j} \hat{Q}_{j}} e^{\frac{i}{\hbar} x_{j} \hat{P}^{j}},
$$

or

$$
W_{2}(x, \alpha)=e^{\frac{i}{\hbar} x_{j} \hat{P}^{j}} e^{\frac{i}{\hbar} \alpha^{j} \hat{Q}_{j}} .
$$

These various forms differ by multiplication by a complex number of modulus one and give rise to different "orderings" (see [1]).

If we select a complex structure on $V$, defined by a tensor $J: V \rightarrow V$ satisfying $J^{2}=-\mathbb{I}$, we can define two operators 


$$
a(v)=\frac{1}{\sqrt{2}}(R(v)+i R(J v)) \quad a^{+}(v)==\frac{1}{\sqrt{2}}(R(v)-i R(J v)),
$$

which will be called annihilation and creation operators respectively. Also the natural symplectic structure $\omega=d \alpha^{j} \wedge d x_{j}$ can be written in the complex coordinates

as

$$
z_{j}=\frac{1}{\sqrt{2}}\left(x_{j}+i \alpha^{j}\right) \quad z_{j}^{*}=\frac{1}{\sqrt{2}}\left(x_{j}-i \alpha^{j}\right)
$$

$$
\omega=i d z_{j}^{*} \wedge d z_{j}
$$

Trivially it follows that $\left(z_{1}, \cdots, z_{n}, \cdots\right)$ are coordinates of a Lagrangian subspace, as well as $\left(z_{1}^{*}, \cdots, z_{n}^{*}, \cdots\right)$.

In these coordinates, we have the Weyl map given by

$$
z \mapsto D(z)=e^{z a^{+}-z^{*} a} .
$$

In this form, Weyl operators are called displacement operators and are used to construct "coherent states".

By means of a symplectic linear transformation, $T: V \rightarrow V$ such that $\omega\left(T v_{1}, T v_{2}\right)=$ $\omega\left(v_{1}, v_{2}\right)$, we may define an automorphism on the set of unitary operators by setting:

$$
W(T v)=\nu_{T}(W(v))=U(T) W(v) U^{\dagger}(T),
$$

because automorphisms of the unitary group are inner.

Thus with a one-parameter group of linear homogeneous symplectic transformations, we define a one-parameter group of unitary transformations. As symplectic linear transformations have an infinitesimal generator associated with a quadratic function on $V$, the corresponding infinitesimal generator of the unitary transformation (Stone's theorem) shall be the operator associated with the quadratic function on $V$. In conclusion we obtain:

Lemma 1. We can associate self-adjoint operators with quadratic functions on $V$.

By using the set of complex coordinates introduced above, and the association

$$
z \mapsto W(z)=e^{z a^{+}-z^{*} a},
$$

we can construct the automorphism

$$
A \mapsto A(z)=W^{\dagger}(z) A W(z) .
$$

By using the vacuum state $|0\rangle$ as a fiducial vector, defined for instance as $a|0\rangle=0$, we may set a one-to-one correspondence between Hermitian operators and functions on $V$ by setting:

$$
f_{A}(z)=\langle 0|A(z)| 0\rangle=\langle z|A| z\rangle .
$$

This function is known as the Berezin symbol of the operator $A$ and allows to define an invertible mapping between Hilbert-Schmidt operators and square integrable functions on $V$.

We should also remark that the map

$$
z \mapsto W(z)|0\rangle=|z\rangle
$$

is a way to immerse $\mathbb{C}^{n}$ (the phase space), as a submanifold (or a subset) of the Hilbert space $\mathcal{H}$ (say, the Fock space). But it is not a linear map onto the image. 
If we consider the expectation value functions

$$
e_{A}(\psi)=\frac{\langle\psi|A| \psi\rangle}{\langle\psi \mid \psi\rangle},
$$

we may pull-back them to $\mathbb{C}^{n}$ by considering:

$$
e_{A}(z)=\frac{\langle z|A| z\rangle}{\langle z \mid z\rangle}
$$

Another important point regards the algebraic structures on the sets. As the mapping (24) is one-to-one, it is possible to associate a function to the product of two operators, and thus define a binary operation on functions associated with operators by setting

$$
A \mapsto f_{A}(\psi), B \mapsto f_{B}(\psi) \quad A B \mapsto f_{A B}(\psi)=\langle\psi|A B| \psi\rangle=\left(f_{A} \star f_{B}\right)(\psi) .
$$

When we pull-back this product to the phase space (i.e. define $\left.\left(f_{A} \star f_{B}\right)(z)\right)$, we get a star-product very close to the usual Moyal product ([30]). As a matter of fact

$$
\lim _{\hbar \rightarrow 0} \frac{f_{A} \star f_{B}-f_{B} \star f_{A}}{\hbar}=\left\{f_{A}, f_{B}\right\}
$$

and thus we can recover the Poisson bracket (associated to the symplectic form $\omega$ ) on phase space.

Let us summarize then the results presented so far:

- Weyl maps are applications from a symplectic vector space $(V, \omega)$ to the set of unitary operators of some Hilbert space $U(\mathcal{H})$ satisfying two properties:

- the map is strongly continuous,

- $W\left(v_{1}\right) W\left(v_{2}\right)=W\left(v_{2}\right) W\left(v_{1}\right) e^{\frac{i}{\hbar} \omega\left(v_{1}, v_{2}\right)}$ for any $v_{1}, v_{2} \in V$.

- Stone's theorem allows us to define the infinitesimal generator of the unitary transformation corresponding to the vector $v$. We denote this infinitesimal generator as $i R(v)$ :

$$
W(v)=e^{i R(v)} \quad \forall v \in V .
$$

The condition above implies that

$$
\left[R\left(v_{1}\right), R\left(v_{2}\right)\right]=i \hbar \omega\left(v_{1}, v_{2}\right) \quad \forall v_{1}, v_{2} \in V
$$

- If we realize the symplectic vector space $V$ as the cotangent bundle (with its canonical symplectic form) of some Lagrangian submanifold $L \subset V$, i.e. $V \sim T^{*} L$; we can write the expression of the Weyl map as:

$$
W(x, \alpha)=e^{\frac{i}{\hbar}(x \hat{P}+\alpha \hat{Q})} \quad(x, \alpha) \in T^{*} L ;
$$

where the operators $\hat{P}$ and $\hat{Q}$ via Stone's theorem and have an associated action on the elements of $\mathrm{E}^{2}\left(L, \mu_{L}\right)$ as

$$
(\hat{Q} \psi)(y)=y \psi(y) \quad(\hat{P} \psi)(y)=-i \hbar \frac{\partial \psi}{\partial y} \quad \psi \in \mathrm{E}^{2}\left(L, \mu_{L}\right) .
$$

- We can also introduce the set of complex coordinates $\left(z, z^{*}\right)$ and write the expression of the Weyl map as

$$
W(z)=e^{z a^{+}-z^{*} a} ;
$$

for $a$ and $a^{+}$the creation and annihilation operators 21 . 
3.2. Weyl maps and Poisson tensors. Let us elaborate a little further and consider a similar construction, but having as initial set the Poisson vector space $\left(V^{*}, \Lambda\right)$, i.e. the dual space $V^{*}=\operatorname{Lin}(V, \mathbb{R})$ and the Poisson tensor $\Lambda$ defined from the symplectic form $\omega$ as:

$$
\Lambda\left(\alpha_{v_{1}}, \alpha_{v_{2}}\right)=\omega\left(v_{1}, v_{2}\right),
$$

for $\alpha_{v_{i}}$ the element of $V^{*}$ defined as

$$
\alpha_{v_{i}}(u)=\omega\left(v_{i}, u\right) \quad \forall u \in V .
$$

With these elements, we define the Weyl map as an application

$$
W:\left(V^{*}, \Lambda\right) \rightarrow \mathcal{H},
$$

being strongly continuous, and satisfying the condition

$$
W\left(\alpha_{1}\right) W\left(\alpha_{2}\right)=W\left(\alpha_{2}\right) W\left(\alpha_{1}\right) e^{i \Lambda\left(\alpha_{1}, \alpha_{2}\right)} .
$$

If we use this as our starting point, we may define Weyl systems also for degenerate Poisson structures. This turns out to be very useful when we deal with constrained systems in the sense of Dirac and Bergmann [15.

3.3. Weyl systems and linear transformations. Consider again the symplectic vector space $(V, \omega)$ and a linear transformation $T: V \rightarrow V$. We can define a new tensor:

$$
\omega_{T}\left(v_{1}, v_{2}\right)=\omega\left(T v_{1}, T v_{2}\right) .
$$

If $T$ is invertible, $\omega_{T}$ is again a symplectic form. We can thus associate a Weyl system to the new symplectic form $\omega_{T}$, in the form:

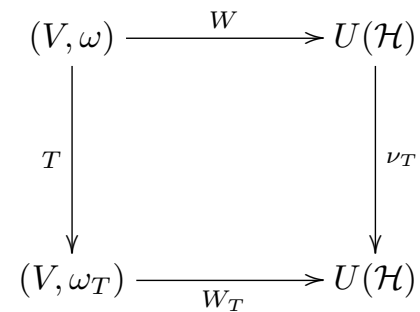

As the diagram is commutative, $\nu_{T}(W(v))=W_{T}(T v)$.

At the infinitesimal level, we have that $R_{T}(v)=R(T v)$ and therefore $R_{T}(v)$ is not Hermitian with respect to the Hermitian structure on $\mathcal{H}$ which makes $W(v)$ unitary. Hence, this is a situation where $R_{T}(v)$ may have a real spectrum without being Hermitian. This situation has been considered in the literature as the one using pseudo-Hermitian operators ([2, 41, 29]).

When $T$ is a symplectic transformation, and hence $\omega_{T}=\omega$, the transformation $\nu_{T}$ is an inner automorphism:

$$
\nu_{T}(W(v))=U_{T}^{\dagger} W(v) U_{T} .
$$

If we consider now a one-parameter family of linear symplectic transformations $T(\lambda)$, where $\lambda \in \mathbb{R}$; we know that the infinitesimal generator of this family is a Hamiltonian vector field. As the transformation is linear, we also know that the associated Hamiltonian function is quadratic. But we can also consider the infinitesimal generator of the associated unitary transformation $U_{T(\lambda)}$. This infinitesimal 
generator will be a self-adjoint operator on $\mathcal{H}$, which is thus associated to the quadratic Hamiltonian function on $V$ which generated the family of linear symplectic transformations.

As an example, if we consider the transformation

$$
T=\left(\begin{array}{cc}
0 & -1 \\
1 & 0
\end{array}\right)
$$

the associated unitary operator is given by the Fourier transform.

Let us consider now the Heisenberg-Weyl Lie algebra $(V \oplus \mathbb{R}, \omega)$ defined as

$$
\left[v_{1}, v_{2}\right]=\omega\left(v_{1}, v_{2}\right)
$$

and its holomorph, i.e. the extension of the algebra with its derivation algebra. Let us recall that the derivation algebra of a Lie algebra is the set of linear transformations $A: V \rightarrow V$ which satisfy:

$$
A\left(\left[v_{1}, v_{2}\right]\right)=\left[A\left(v_{1}\right), v_{2}\right]+\left[v_{1}, A\left(v_{2}\right)\right] .
$$

In the present situation, this corresponds to the algebra of infinitesimal canonical transformations of $V$. If we consider then this extension of the Heisenberg-Weyl algebra, the Weyl construction shows that the entire associated Lie group can be represented by unitary operators on the Hilbert space on which the Weyl operators act.

3.4. Associating operators to more general functions. We have seen that our previous construction is able to provide a unitary operator to represent any function of $V$ which is at most quadratic. What can be said for more general functions?

The trick elaborated by Weyl was the following. Consider the realization $V \sim$ $T^{*} L$ which assigns a pair $(x, \alpha)$ to any element $v \in V$. Now, we can consider the double Fourier transform of a function $f: V \rightarrow \mathbb{R}$ as

$$
\tilde{f}(q, p)=\iint d x d \alpha e^{i(\alpha q+x p)} f(x, \alpha) .
$$

Now define the operator $\hat{f}$ associated to the function $f$ as

$$
\hat{f}(\hat{Q}, \hat{P})=\int d x d \alpha e^{\frac{i}{\hbar}(\alpha \hat{Q}+x \hat{P})} f(x, \alpha) .
$$

This map can be inverted, i.e. we can find a map from operators acting on $\mathcal{H}$ onto functions defined on $V$, such that it is an involutive map of index two. The inverse, namely the function $f_{A}$ associated to an operator $A$ on the Hilbert space, can be written as:

$$
f_{A}(v)=\operatorname{Tr} A W(v)
$$

We might also use the symplectic Fourier transform

$$
\tilde{f}(q, p)=\int d x d \alpha e^{-i(\omega((x, \alpha),(q, p)))} \operatorname{Tr}\left(\hat{A} W^{\dagger}(x, \alpha)\right) .
$$

Definition 1. If we apply this to the density operator associated to a pure state $|\psi\rangle, \rho_{\psi}=|\psi\rangle\langle\psi|$, we obtain:

$$
f_{\rho_{\psi}}(q, p)=\int d \xi e^{\frac{i}{\hbar} p \xi} \psi(q+\xi / 2) \psi^{*}(q-\xi / 2) .
$$

This is called the Wigner function associated to the state $|\psi\rangle$. 
We can conclude thus with the following statement:

Lemma 2. The Wigner-Weyl map defines a bijection between Hilbert-Schmidt operators and square-integrable functions on phase space.

If we also denote by $W$ the map $f \mapsto \hat{f}=W(f)$ (i.e. applied to functions on $V$ ), we can use the bijection to transport the associative product of the $\mathbb{C}^{*}$-Weyl algebra generated by unitary operators on $\mathcal{H}$ onto the set of functions on $V$. This defines the $\star$-product we saw above:

$$
(f \star g)(x, \alpha)=W^{-1}(W(f) . W(g))(x, \alpha)=\operatorname{Tr}\left(W(f) W(g) W^{\dagger}(x, \alpha)\right) .
$$

This is the integral form of the Moyal product, which is usually written in terms of bidifferential operators

$$
(f \star g)(q, p)=f(q, p) e^{i \frac{\hbar}{2}\left(\frac{\overleftarrow{\partial}}{\partial q} \frac{\vec{\partial}}{\partial p}-\frac{\overleftarrow{\partial}}{\partial p} \frac{\vec{\partial}}{\partial q}\right)} g(q, p)
$$

or equivalently

$$
(f \star g)(q, p)=f\left(q+i \frac{\hbar}{2} \frac{\vec{\partial}}{\partial p}, p-i \frac{\hbar}{2} \frac{\vec{\partial}}{\partial q}\right) g(q, p) .
$$

It should be clear now that all descriptions in terms of operators have a counterpart in terms of complex valued functions on the phase space with the Moyal product. For instance, we can write the eigenvalue problem for the Hamiltonian of our quantum system as:

$$
H \star \rho_{E}=E \rho_{E},
$$

where $H$ is the function corresponding to the Hamiltonian operator and $\rho_{E}$ is the function representing the eigenstate with energy $E$. Similarly we can write evolution equations and their exponentiation as a Taylor expansion in terms of $\star-$ products.

3.5. Tomograms. The Wigner function associated to a state $\psi$ was introduced in 1932 as a tool for the study of quantum corrections to classical equilibrium distributions 42. However, unlike the distribution function of classical statistical mechanics which is a probability distribution function on phase space, the Wigner function is only a quasi-distribution, i.e. it is not positive definite.

The tomogram description of quantum mechanics uses a formulation in which the quantum state is described by conventional non-negative probability distributions. Thus, within this formulation both the classical and the quantum descriptions of a physical system use true probability distributions, and this property makes easier to compare predictions of the two descriptions. Moreover, it makes also easier to deal with the quantum-classical transition because the describing functions are defined on the same carrier space.

We shall not enter in this fascinating subject, but instead refer to some previous work to find details on this formulation in the same spirit of these notes [24, 23.

\section{The geometrical Description of Quantum Mechanics}

4.1. The Hermitian structure. To consider Quantum Mechanics from a differential geometric point of view, it is convenient to consider the Hilbert space $\mathcal{H}$ as a real differential manifold. In order to do this, we have to convert the Hermitian inner product $\langle\cdot, \cdot\rangle$ which defines $\mathcal{H}$ into an Hermitian tensor. This is quite similar to what we do in the transition from special relativity to general relativity. In that 
case, the Minkowski product $\eta_{\mu \nu} x^{\mu} x^{\nu}$ is replaced by the tensor $\eta_{\mu \nu} d x^{\mu} \otimes d x^{\nu}$ and thus space-time becomes a manifold whose tangent space becomes endowed with a Minkowskian product. In the present case, the Hilbert space $\mathcal{H}$ is replaced by a Hilbertian manifold, whose tangent space is a Hilbert space with the inner product $\langle\cdot, \cdot\rangle$.

To be definite, let us consider an orthonormal basis $\left\{\left|e_{j}\right\rangle\right\}_{j=1, \ldots}$ for $\mathcal{H}$ and define the coordinate functions:

$$
\left\langle e_{j} \mid \psi\right\rangle=z^{j}(\psi)=q^{j}(\psi)+i p_{j}(\psi)
$$

where $q^{j}$ and $p_{j}$ are real functions. With this in mind, we replace the inner product $\langle\psi \mid \psi\rangle$ by $\langle d \psi \otimes d \psi\rangle$, where $d \psi$ is a Hilbert-space valued one-form, i.e. a map from $T \mathcal{H}$ to $\mathcal{H}$ linear along the fibers:

$$
\sum_{j}\left|e_{j}\right\rangle\left\langle e_{j} \mid d \psi\right\rangle=\sum_{j} d z^{j}\left|e_{j}\right\rangle
$$

Then the expression of the product becomes:

$$
\langle d \psi \otimes d \psi\rangle=d \bar{z}^{j}\left\langle e_{j} \mid e_{k}\right\rangle d z^{k}=d \bar{z}^{j} \otimes d z^{k}\left\langle e_{j} \mid e_{k}\right\rangle
$$

Now using the real functions $q^{j}, p_{j}$ introduced above we get:

$$
\begin{aligned}
\langle d \psi \otimes d \psi\rangle= & \left(d q^{j}-i d p_{j}\right) \otimes\left(d q^{k}+i d p_{k}\right)\left\langle e_{j} \mid e_{k}\right\rangle= \\
& \left(d q^{j} \otimes d q^{k}+d p_{j} \otimes d p_{k}+i\left(d q^{j} \otimes d p_{k}-d p_{j} \otimes d q^{k}\right)\right)\left\langle e_{j} \mid e_{k}\right\rangle
\end{aligned}
$$

Because of the orthonormality of the base we find:

$$
\langle d \psi \otimes d \psi\rangle=\left(d q^{j} \otimes d q^{k}+d p_{j} \otimes d p_{k}+i\left(d q^{j} \otimes d p_{k}-d p_{j} \otimes d q^{k}\right)\right) \delta_{j k}
$$

We can also consider the scalar product on the dual space $\mathcal{H}^{*}$ and thus introduce two tensors:

$$
G=\frac{\partial}{\partial q^{k}} \otimes \frac{\partial}{\partial q_{k}}+\frac{\partial}{\partial p^{k}} \otimes \frac{\partial}{\partial p_{k}}
$$

and

$$
\Lambda=\frac{\partial}{\partial p_{k}} \otimes \frac{\partial}{\partial q^{k}}-\frac{\partial}{\partial q^{k}} \otimes \frac{\partial}{\partial p_{k}} .
$$

Clearly,

$$
\Lambda(d f, d g)=\frac{\partial f}{\partial p_{k}} \otimes \frac{\partial g}{\partial q^{k}}-\frac{\partial f}{\partial q^{k}} \otimes \frac{\partial g}{\partial p_{k}}
$$

defines a Poisson braket on the space of functions while

$$
G(d f, d g)=\frac{\partial f}{\partial q^{k}} \otimes \frac{\partial g}{\partial q_{k}}+\frac{\partial f}{\partial p^{k}} \otimes \frac{\partial g}{\partial p_{k}}
$$

defines a commutative bracket.

And we can define the Hermitian bracket as a combination of the above products:

$$
\langle d f, d g\rangle=G(d f, d g)+i \Lambda(d f, d g) .
$$


4.2. Other geometrical objects. We may use the set of real coordinate functions introduced in the previous section to write explicitly the complex structure of $\mathcal{H}$. We know that the Hilbert space is complex, therefore if we want to describe it as a real differentiable manifold, we need to identify the corresponding complex structure. It corresponds to a $(1,1)$-tensor $J$, satisfying the property $J^{2}=-\mathbb{I}$, which written in the coordinates above has the form

$$
J=\sum_{k}\left(d q^{k} \otimes \frac{\partial}{\partial p_{k}}-d p_{k} \otimes \frac{\partial}{\partial q^{k}}\right) .
$$

The other ingredient of the Hilbert space structure of $\mathcal{H}$ is its linear structure. This also can be encoded in a tensor, a vector field in this case, which is the dilation vector field $\Delta: \mathcal{H} \rightarrow \mathcal{H} \times \mathcal{H} \sim T \mathcal{H}$. It is defined as $\Delta(\psi)=(\psi, \psi)$ and hence takes the coordinate expression:

$$
\Delta(\psi)=q^{k} \frac{\partial}{\partial q^{k}}+p_{k} \frac{\partial}{\partial p_{k}}
$$

The complex and the linear structures can be thus combined as

$$
J(\Delta)=p_{k} \frac{\partial}{\partial q^{k}}-q^{k} \frac{\partial}{\partial p_{k}},
$$

which is the infinitesimal generator of the multiplication by a phase.

Let us go back now to the problem of the star product at the level of the functions. Consider an operator $A$ on $\mathcal{H}$ and the evaluation functions

$$
f_{A}(\psi)=\langle\psi|A| \psi\rangle,
$$

and the expectation value function

$$
e_{A}(\psi)=\frac{\langle\psi|A| \psi\rangle}{\langle\psi \mid \psi\rangle} .
$$

The $\star$-product which translates the associative algebra structure of the set of operators reads

$$
f_{A} \star f_{B}=\frac{1}{2} G\left(d f_{A}, d f_{B}\right)+\frac{i}{2} \Lambda\left(d f_{A}, d f_{B}\right)=f_{A B} .
$$

and

$$
e_{A} \star e_{B}=e_{A} e_{B}+\frac{1}{2} G_{P}\left(d e_{A}, d e_{B}\right)+\frac{i}{2} \Lambda_{P}\left(d e_{A}, d e_{B}\right)=e_{A B},
$$

where $G_{P}=\langle\Delta \mid \Delta\rangle G$ and $\Lambda_{P}=\langle\Delta \mid \Delta\rangle$ (we shall go back to these objects in the next sections).

Definition 2. A Kählerian function is a function $f$ such that the Hamiltonian vector field $\Gamma_{f}, \Lambda(d f)=\Gamma_{f}$, is also a Killing vector field, i.e.

$$
L_{\Gamma_{f}} G=0
$$

On Kählerian functions, $G$ defines a Jordan algebra structure, while $\Lambda$ defines a Lie structure. Both structures are compatible (they define a structure called Lie-Jordan algebra [16, 22]) and together define the $\mathbb{C}^{*}$-algebra corresponding to bounded operators.

The operator norm can also be obtained from the function as $\|A\|=\sup _{A}(\psi)$ for any positive self-adjoint operator $A$ and any normalized vector $\psi$. 
If $A$ is a general, possibly non self-adjoint operator, we have that $\|A\|^{2}=\left\|A^{*} A\right\|$ and this is translated as

$$
\|A\|^{2}=\sup _{\psi}\left(\bar{f}_{A} \star f_{A}\right)(\psi)
$$

Remark 1. The functional representation of a $\mathbb{C}^{*}$-algebra requires a uniform Kähler bundle $[10,9,8, p: \mathcal{P} \rightarrow \mathcal{B}$. $\mathcal{P}$ will be the set of pure states of $\mathcal{A}$, while $\mathcal{B}$ is the spectrum of $\mathcal{A}$ (i.e. the set of unitary equivalent classes of irreducible Hilbertian representations of $\mathcal{A}$ ). $p$ is the natural projection, associating the equivalence class of irreducible representations containing the GNS representation induced by the pure state $\omega$ :

$$
\omega_{\psi}(A)=e_{\pi(A)}(\psi)=\operatorname{Tr} \omega_{\psi} \pi(A) \quad \forall A \in \mathcal{A}
$$

For further details on the geometrical interpretation of the GNS construction see Chruchinski-Marmo [7].

Let us consider now the relation with the notion of Gelfand transform. The Gelfand transform of an operator $A$ is defined as

$$
e_{A}: \mathcal{P} \rightarrow \mathbb{C} \quad \mathcal{P} \ni \omega \mapsto e_{A}(\omega)=\omega(A)
$$

The Gelfand transform $A \mapsto e_{A}$ is a linear involutive preserving injection of $\mathcal{A}$ into $\mathcal{F}(\mathcal{P})$. For $A, B \in A, e_{A B}=e_{A} \star e_{B}$ and

$$
\|A\|^{2}=\sup _{\omega \in \mathcal{P}}\left(\bar{e}_{A} \star e_{A}\right)(\omega) .
$$

The range of the transform is the set

$$
\mathcal{K}_{u}(\mathcal{P})=\{f \in \mathcal{K} \mid f, \bar{f} \star f, f \star \bar{f} \text { are uniformly continuous on } \mathcal{P}\}
$$

4.3. Projective Hilbert spaces as Kähler manifolds. We already saw above that from a physical point of view, the probabilistic interpretation requires that the set of states of a system is not a vector space but a complex projective one. Our aim now is to present the geometrical structures arising in this case.

First let us study the expression of the Hermitian tensor in $\mathcal{P H}$. It is simple to obtain a Hermitian tensor field on $\mathcal{H}$ which vanishes on vertical vector fields (generated by $\Delta$ and $J(\Delta)$ ) and provides a projectable function when evaluated on projectable vector fields:

$$
\frac{\langle d \psi \otimes d \psi\rangle}{\langle\psi \mid \psi\rangle}-\frac{\langle\psi \mid d \psi\rangle \otimes\langle d \psi \mid \psi\rangle}{\langle\psi \mid \psi\rangle^{2}}
$$

From a geometrical point of view, the complex projective space is obtained as the quotient manifold obtained from the Hilbert space $\mathcal{H}$ with respect to the foliation generated by two vector fields:

- the dilation vector field $\Delta=q^{k} \frac{\partial}{\partial q^{k}}+p_{k} \frac{\partial}{\partial p_{k}}$, and

- the phase-change vector field obtained as the vector field resulting from the action of the complex structure $J$ on $\Delta$, i.e. $\Gamma=J(\Delta)=q^{k} \frac{\partial}{\partial p_{k}}-$ $p_{k} \frac{\partial}{\partial q^{k}}$. This vector field is the Hamiltonian vector field (with respect to the canonical Poisson structure $\Lambda$ of the function $f(q, p)=\langle\psi \mid \psi\rangle$, associated with the unity operator $\mathbb{I}$..

From this observation it is simple now to study, from a geometrical point of view, the projection of the contravariant tensors introduced on $\mathcal{H}$ to the complex projective space. We need to consider contravariant tensors and functions which are invariant under the action of $\Gamma$ and $\Delta$. 
From this perspective, it is simple to see that the functions $e_{A}$ that we have associated with operators $A$ are trivially projectable. On the other hand, it is simple to see (from the coordinate expressions, for instance) that the tensors $G$ and $\Lambda$ are homogeneous of degree -2 , and hence non-projectable. The homogeneity consideration is useful, though, to modify them by a conformal factor and make them projectable. Thus the objects

$$
G_{p}=\langle\Delta \mid \Delta\rangle G \quad \Lambda_{p}=\langle\Delta \mid \Delta\rangle \Lambda
$$

define projectable tensors.

Particularly the issue on the Poisson tensor has some deep implications from a geometrical point of view.

We may use the two tensors above to realize the $\mathbb{C}^{*}$-algebra of operators on $\mathcal{H}$ as a $\mathbb{C}^{*}$-algebra of functions on the complex projective space. With the help of these functions we can formulate the eigenvalue problem on the complex projective space considered as a real manifold, and write the equations of motion corresponding to the Heisenberg formulation of the evolution equations. Thanks to the Wigner's theorem, it is possible to solve the equations of the motion on the Hilbert space, by exponentiation for instance, and then to project the result onto the ray space to obtain the desired solutions. More likely it is because of this property that physicists have not bothered dealing with Quantum Mechanics on the ray space considered as a real manifold.

4.4. The momentum mapping. To end this quick description of the basics of the geometrical description of Quantum Mechanics, we will now consider another important aspect, here worked out for a specific example.

Given the Hilbert space $\mathcal{H} \equiv \mathbb{C}^{3}$ (or equivalently the corresponding projective space $\mathcal{P} \mathcal{H})$, we know that there is an action of the unitary group $U(3)$ on them. With respect to the canonical symplectic structure of $\mathcal{H}$ this action is strongly symplectic ([26]), and therefore admits a momentum map

$$
\mu: \mathcal{H}_{0}=\mathcal{H}-\{0\} \rightarrow \mathfrak{u}^{*}(3)
$$

or equivalently

$$
\tilde{\mu}: \mathcal{P} \mathcal{H} \rightarrow \mathfrak{u}^{*}(3)
$$

These mappings commute with respect to the natural projection $\pi: \mathcal{H} \rightarrow \mathcal{P H}$, as

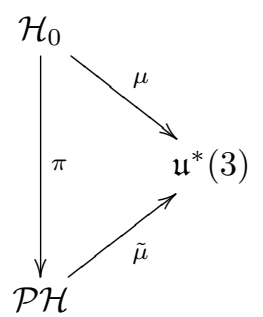

Given a point $|\psi\rangle \in \mathcal{H}$, its image by $\mu$ is an element of the dual of the algebra of operators. From that point of view, it is easy to construct a pairing between states and operators defined as

$$
\mu(\psi)(A)=\langle\psi|A| \psi\rangle=\rho_{\psi}(A)=\operatorname{Tr}\left(A \rho_{\psi}\right) \equiv \operatorname{Tr}(A|\psi\rangle\langle\psi|)
$$


Thus we define the momentum mappings as

$$
\mu(\psi) \equiv \rho_{\psi}=|\psi\rangle\langle\psi| \quad \tilde{\mu}(\psi) \equiv \rho_{[\psi]}=\frac{|\psi\rangle\langle\psi|}{\langle\psi \mid \psi\rangle},
$$

where $|\psi\rangle\langle\psi|$ is the mapping defined on $\mathcal{H}$ as

$$
|\psi\rangle\langle\psi|: \mathcal{H} \rightarrow \mathcal{H} \quad \mathcal{H} \ni \phi \mapsto\langle\psi \mid \phi\rangle| \psi\rangle .
$$

Clearly the mapping $\tilde{\mu}$ is one-to-one. This implies that the geometric structures that we know on $\mathcal{P H}$ must have an analogue on $\mathfrak{u}^{*}(3)$. Indeed, it is simple to see that the geometric structures correspond to the canonical structures of the coadjoint orbit of $U(3)$ on $\mathfrak{u}^{*}(3)$ which contains $\tilde{\mu}([\psi])$.

One important fact is the isomorphism $\mathfrak{u}(3) \rightarrow \mathfrak{u}^{*}(3)$ given by the Killing-Cartan metric. Indeed, there is a one-to-one correspondence between the elements of the Lie algebra and the elements of its dual and this correspondence intertwines the adjoint and the coadjoint actions. We shall denote as $\alpha_{A}$ the element of $\mathfrak{u}^{*}(3)$ corresponding to the observable $i A$ for $A \in \mathfrak{u}(3)$. Besides, as the set of physical observables in this case corresponds to the set of linear functions on $\mathfrak{u}^{*}(3)\left(\mathfrak{u}(\mathcal{H})=\operatorname{Lin}\left(\mathfrak{u}^{*}(\mathcal{H}), \mathbb{R}\right)\right)$, we can use the pullback $\mu^{*}: \mathcal{F}\left(\mathfrak{u}^{*}(3)\right) \rightarrow \mathcal{F}(\mathcal{H})$ to relate the set of Hermitian operators and the set of functions defined on the Hilbert space. The geometrical structures are also related, defining suitable morphisms:

Proposition 1. $\quad$ i) The momentum map is equivariant with respect to the action of $U(\mathcal{H})$ on $\mathcal{H}_{0}$ and the coadjoint action of $U(\mathcal{H})$ on $\mathfrak{u}^{*}(\mathcal{H})$. In particular, this says that the Schrödinger equation of motion on $\mathcal{H}$ is $\mu$-related with the Heisenberg equation of motion on $\mathfrak{u}(\mathcal{H})$ (the space $\mathfrak{u}(\mathcal{H})$ is identified with the dual by means of the scalar product defined by the trace). Moreover,

ii) $\mu^{*}(\hat{A})=f_{A}, \tilde{\mu}^{*}(\hat{A})=e_{A}$.

iii) $\mu^{*}(\{\hat{A}, \hat{B}\})=\left\{f_{A}, f_{B}\right\}$ and $\tilde{\mu}^{*}(\{\hat{A}, \hat{B}\})=\left\{e_{A}, e_{B}\right\}$, where

iv) $\mu^{*}(R(d \hat{A}, d \hat{B}))=G\left(\mu^{*}(d \hat{A}), \mu^{*}(d \hat{B})\right)$ and for the other mapping

$$
\tilde{\mu}^{*}(R(d \hat{A}, d \hat{B}))=G_{P}\left(\tilde{\mu}^{*}(d \hat{A}), \tilde{\mu}^{*}(d \hat{B})\right)+e_{A} e_{B},
$$

where $R$ is the Jordan tensor defined on $\mathfrak{u}^{*}(3)$ from the canonical one on $\mathfrak{u}(3)$ as

$$
\mathcal{R}(\xi)(d \hat{A}, d \hat{B})=\xi\left([A, B]_{+}\right)=\frac{i}{2} \operatorname{Tr} \xi(A B+B A),
$$

for $\xi \in \mathfrak{u}^{*}(3)$, and $\hat{A}$ and $\hat{B}$ are arbitrary elements of $\mathfrak{u}^{*}(3)$.

Proof. Direct computation.

Instead of insisting with additional general aspects of the geometrical formulation of Quantum Mechanics (further details may be found in [5, 6, we shall consider now some specific examples which will illustrate the general picture.

4.5. States: Density states. We have seen how the momentum map $\tilde{\mu}$ allows us to embed the complex projective space $\mathcal{P H}$ on the dual of the Lie algebra $\mathfrak{u}(\mathcal{H})$. The resulting elements represent the set of pure states of the quantum system. But in many physical situations we have more general states, i.e. density states which are convex combinations of pure states. They are represented by a family $\rho=\left\{\rho_{1}, \cdots, \rho_{k}\right\}$, each element satisfying

$$
\rho_{k}^{2}=\rho_{k}, \quad \rho_{k}^{+}=\rho_{k}, \quad \operatorname{Tr} \rho_{k}=1,
$$


along with a probability vector, namely $\vec{p}=\left(p_{1}, p_{2}, \cdots, p_{k}\right)$ with $\sum_{j} p_{j}=1$ and $p_{j} \geq 0 \quad \forall j$. Out of these we construct a density state $\rho=\sum_{j} p_{j} \rho_{j}$. The evaluation of this state on some observable $A$ is given by

$$
\rho(A)=\sum_{j} p_{j} \operatorname{Tr} \rho_{j} A=\operatorname{Tr} \rho A .
$$

We shall call density states all convex combinations of pure states, and we denote them by $\mathcal{D}(\mathcal{H})$ 20, 11].

As any of the elements in $\rho$ can be embedded into $\mathfrak{u}^{*}(\mathcal{H})$, it makes perfect sense to consider $\rho$ also as an element in the dual of the unitary algebra. And we hence consider the geometric structure we defined on $\mathfrak{u}^{*}(\mathcal{H})$ as the Poisson or the Jordan brackets

$$
\begin{aligned}
\left\{f_{A}, f_{B}\right\}(\rho) & =\sum_{k} p_{k} f_{[A, B]_{-}}\left(\psi_{k}\right)=\sum_{k} p_{k}\left\{f_{A}, f_{B}\right\}\left(\psi_{k}\right) \\
\left(f_{A}, f_{B}\right)(\rho) & =\sum_{k} p_{k}\left(f_{A}, f_{B}\right)\left(\psi_{k}\right)
\end{aligned}
$$

where $f_{A}(\rho)=\sum_{k} p_{k} f_{A}(\psi)$.

As for the geometric structures on $\mathcal{D}(\mathcal{H})$ we shall consider it as a real manifold with boundary embedded into the real vector space $\mathfrak{u}^{*}(\mathcal{H})$. On this space the two structures above (36), define a Poisson and a "Riemannian" structure. The Poisson structure is degenerate. However it is also possible to define a generalized complex structure satisfying

$$
J^{3}=-J
$$

The boundary is a stratified manifold, corresponding to the union of symplectic orbits of $U(\mathcal{H})$ of different dimensions, passing through density matrices of not maximal rank. For further information see [20, 21, 25].

\section{EXample I: TWo LeVel QuANtum Systems}

5.1. The Hilbert space. For a two levels system we shall consider an orthonormal basis on $\mathbb{C}^{2}$, say $\left\{\left|e_{1}\right\rangle,\left|e_{2}\right\rangle\right\}$. We introduce thus a set of coordinates

$$
\left\langle e_{j} \mid \psi\right\rangle=z^{j}(\psi)=q^{j}(\psi)+i p_{j}(\psi) \quad j=1,2 .
$$

In the following we shall use $z^{j}$ or $q^{j}, p_{j}$ omitting the dependence in the state $\psi$ as it is usually done in differential geometry.

The set of physical states is not equal to $\mathbb{C}^{2}$, since we have to consider the equivalence relation given by the multiplication by a complex number i.e.

$$
\psi_{1} \sim \psi_{2} \Leftrightarrow \psi_{2}=\lambda \psi_{1} \quad \lambda \in \mathbb{C}_{0}=\mathbb{C}-\{0\} .
$$

And besides, the norm of the state must be equal to one. These two properties can be encoded in the following diagram:

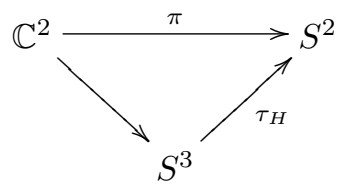

where $S^{2}$ and $S^{3}$ stand for the two and three dimensional spheres, and the projection $\tau_{H}$ is the Hopf fibration. The projection $\pi$ is associating each vector with the one-dimensional complex vector space to which it belongs. Thus we see how this 
projection factorizes through a projection onto $S^{3}$ and a further projection given by the Hopf fibration (a $U(1)$-fibration).

The Hermitian inner product on $\mathbb{C}^{2}$ can be written in the coordinates $z_{1}, z_{2}$ as

$$
\langle\psi \mid \psi\rangle=\bar{z}_{j} z^{k}\left\langle e_{k} \mid e_{j}\right\rangle=\bar{z}_{j} z^{j}
$$

Equivalently we can write it in real coordinates $q, p$ and obtain:

$$
\langle\psi \mid \psi\rangle=p_{1}^{2}+p_{2}^{2}+q_{1}^{2}+q_{2}^{2}
$$

The associated tensor field reads

$$
\langle d \psi \otimes d \psi\rangle=d \bar{z}_{j} \otimes d z^{k}\left\langle e_{k} \mid e_{j}\right\rangle
$$

or in real coordinates

$$
\langle d \psi \otimes d \psi\rangle=\left(d q_{j}-i d p^{j}\right) \otimes\left(d q^{k}+i d p_{k}\right)\left\langle e_{k} \mid e_{j}\right\rangle .
$$

This expression can be decomposed into its real and its imaginary parts and obtain

$$
d q_{k} \otimes d q^{k}+d p^{k} \otimes d p_{k} \quad d q_{j} \otimes d p^{j}-d p^{j} \otimes d q_{j}=d q_{j} \wedge d p^{j} .
$$

Hence we recognize a Riemannian structure, on the left (the real part) and a symplectic structure (the imaginary one, on the right).

We can also obtain these tensors in contravariant form if we take as starting point the Hilbert space $\left(\mathbb{C}^{2}\right)^{*}$. If we repeat the steps above, we obtain the two contravariant tensors:

$$
G=\frac{\partial}{\partial q_{k}} \otimes \frac{\partial}{\partial q^{k}}+\frac{\partial}{\partial p^{k}} \otimes \frac{\partial}{\partial p_{k}} \quad \Lambda=\frac{\partial}{\partial q_{k}} \wedge \frac{\partial}{\partial p^{k}} .
$$

Other tensors encode the complex vector space structure of $\mathcal{H}=\mathbb{C}^{2}$ :

- the dilation vector field $\Delta=q_{1} \frac{\partial}{\partial q_{1}}+p^{1} \frac{\partial}{\partial p_{1}}+q_{2} \frac{\partial}{\partial q_{2}}+p^{2} \frac{\partial}{\partial p_{2}}$,

- and the complex structure tensor $J=d p_{1} \otimes \frac{\partial}{\partial q_{1}}-d q^{1} \otimes \frac{\partial}{\partial p^{1}}+d p_{2} \otimes \frac{\partial}{\partial q_{2}}-$ $d q^{2} \otimes \frac{\partial}{\partial p^{2}}$.

By combining both tensors, we can define the infinitesimal generator of the multiplication by a phase:

$$
\Gamma=J(\Delta)=p_{1} \frac{\partial}{\partial q_{1}}-q^{1} \frac{\partial}{\partial p^{1}}+p_{2} \frac{\partial}{\partial q_{2}}-q^{2} \frac{\partial}{\partial p^{2}} .
$$

Thus we see how $\Delta$ is responsible for the quotienting from $\mathbb{C}_{0}^{2}$ onto $S^{3}$, while $\Gamma$ is responsible for the Hopf fibration $S^{3} \rightarrow S^{2}$.

By using the Hermitian operators $\left\{\sigma_{0}, \sigma_{1}, \sigma_{2}, \sigma_{3}\right\}$ to construct functions $\langle\psi|A| \psi\rangle$, we obtain the real quadratic functions

$$
q_{1}^{2}+p_{1}^{2}+q_{2}^{2}+p_{2}^{2}, \quad q_{1} q_{2}+p_{1} p_{2}, \quad q_{1} p_{2}-p_{1} q_{2}, \quad q_{1}^{2}+p_{1}^{2}-\left(q_{2}^{2}+p_{2}^{2}\right) .
$$

It is not difficult now to compute the Poisson brackets of these quadratic functions to find that they are the Hamiltonian for the infinitesimal generators of the $\mathfrak{u}(2)$ algebra. We may also compute explicitly the Jordan brackets, as for instance

$$
\left\{q_{1}^{2}+p_{1}^{2}+q_{2}^{2}+p_{2}^{2}, q_{1} q_{2}+p_{1} p_{2}\right\}_{+}=4\left(q_{1} q_{2}+p_{1} p_{2}\right) .
$$

Similar results are obtained with the other brackets. The result we want to point out is

Lemma 3. The function $q_{1}^{2}+p_{1}^{2}+q_{2}^{2}+p_{2}^{2}$ acts, with respect to the Jordan bracket, as the identity operator except for a normalization factor. 
We also find

$$
\left\{q_{1} q_{2}+p_{1} p_{2}, q_{1} q_{2}+p_{1} p_{2}\right\}_{+}=4\left(q_{1}^{2}+p_{1}^{2}+q_{2}^{2}+p_{2}^{2}\right) .
$$

And analogously for the other quadratic functions. We have

Lemma 4. The product of all functions (in the family above) with themselves produce a multiple of the quadratic isotropic function.

We also can obtain easily

$$
\left\{q_{1} q_{2}+p_{1} p_{2}, q_{1} p_{2}-p_{1} q_{2}\right\}_{+}=0=\left\{q_{1} q_{2}+p_{1} p_{2}, q_{1}^{2}+p_{1}^{2}-\left(q_{1}^{2}+p_{1}^{2}\right)\right\}_{+}
$$

The additional relevant property is that the Hamiltonian vector field are Killing vectors. In terms of brackets this amounts to:

$$
\left\{f,\{g, h\}_{+}\right\}=\{\{f, g\}, h\}_{+}+\{g,\{f, h\}\}_{+}
$$

This condition, plus the compatibility between the associator and the Poisson bracket implies that the two brackets combined define a Lie-Jordan algebra.

In particular, by considering generic quadratic functions of two complex coordinates, we find a complex valued quadratic function whose real and imaginary parts are quadratic functions of the previous type. All in all, the result is:

Lemma 5. Complex valued quadratic functions close on a $\mathbb{C}^{*}$-algebra with respect to the Hermitian bracket.

Thus we have found that Hermitian operators are associated with Hamiltonian vector fields which are also Killing. As a matter of fact, this property characterizes functions on $\mathbb{C}^{2}$ which are associated with Hermitian operators.

5.2. The projective space. We can also consider the Hermitian tensor field related to the one on the complex projective space. It is important to remark that while forms can not be projected, contravariant tensor fields can. This is the reason why we introduced the contravariant tensors $\Lambda$ and $G$. Thus by considering

$$
G=\frac{\partial}{\partial q^{k}} \otimes \frac{\partial}{\partial q_{k}}+\frac{\partial}{\partial p_{k}} \otimes \frac{\partial}{\partial p_{k}},
$$

we can define a projectable tensor as:

$$
\tilde{G}=\left(q_{1}^{2}+q_{2}^{2}+p_{1}^{2}+p_{2}^{2}\right)\left(\frac{\partial}{\partial q^{k}} \otimes \frac{\partial}{\partial q_{k}}+\frac{\partial}{\partial p_{k}} \otimes \frac{\partial}{\partial p_{k}}\right)
$$

Because computations on the vector space are easier to carry on with respect to computations on the complex projective space, it is convenient to consider tensors on $\mathcal{H}$ which reproduce the same results that we would have on the complex projective space. Thus we want a tensor $\tilde{G}_{H}$ defined by

$$
\tilde{G}_{H}\left(\pi^{*} d f, \pi^{*} d g\right)=\tilde{G}\left(\pi^{*} d f, \pi^{*} d g\right)
$$

and identically vanishing on

$$
\langle\psi \mid d \psi\rangle=q_{j} d q^{j}+p^{j} d p_{j}+i\left(q_{j} d p^{j}-p^{j} d q_{j}\right) .
$$

The tensor is simple to construct as

$$
\tilde{G}_{H}=\tilde{G}-(\Delta \otimes \Delta+\Gamma \otimes \Gamma)-i(\Delta \otimes \Gamma-\Gamma \otimes \Delta) .
$$

Analogously we can introduce

$$
\tilde{\Lambda}_{H}=\left(q_{1}^{2}+q_{2}^{2}+p_{1}^{2}+p_{2}^{2}\right)\left(\frac{\partial}{\partial q_{j}} \wedge \frac{\partial}{\partial p^{j}}\right)
$$


The next step is to consider the projectable quadratic functions. If we consider the basis of the Hermitian operators given by the Pauli matrices, we find:

$$
\begin{gathered}
e_{\sigma_{0}}=1 \quad e_{\sigma_{1}}=\frac{q_{1} q_{2}+p_{1} p_{2}}{q_{1}^{2}+q_{2}^{2}+p_{1}^{2}+p_{2}^{2}} \\
e_{\sigma_{2}}=\frac{q_{1} p_{2}-p_{1} q_{2}}{q_{1}^{2}+q_{2}^{2}+p_{1}^{2}+p_{2}^{2}} \quad e_{\sigma_{3}}=\frac{q_{1}^{2}+p_{1}^{2}-q_{2}^{2}-p_{2}^{2}}{q_{1}^{2}+q_{2}^{2}+p_{1}^{2}+p_{2}^{2}}
\end{gathered}
$$

We find that only the functions associated with $\left\{\sigma_{1}, \sigma_{2}, \sigma_{3}\right\}$ define non-trivial functions on the complex projective space. Of course, their associated vector fields generate the algebra of $S U(2)$.

We can compute now the action of the tensor $\tilde{G}$ on these functions, and we obtain:

$$
\begin{gathered}
\tilde{G}\left(e_{\sigma_{0}}, f\right)=0 \quad \forall f \\
\tilde{G}\left(e_{\sigma_{1}}, e_{\sigma_{1}}\right)=e_{0}-4 e_{\sigma_{1}}^{2} \\
\tilde{G}\left(e_{\sigma_{2}}, e_{\sigma_{2}}\right)=e_{0}-4 e_{\sigma_{2}}^{2} \\
\tilde{G}\left(e_{\sigma_{3}}, e_{\sigma_{3}}\right)=4\left(e_{0}-e_{3}^{2}\right) \\
\tilde{G}\left(e_{\sigma_{1}}, e_{\sigma_{2}}\right)=-4\left(e_{\sigma_{1}} e_{\sigma_{2}}\right)
\end{gathered}
$$

In an analogous way, other products can be computed. But we already see that, due to the denominator, we do not find "orthogonality" of the expectation values associated with different Pauli matrices. Instead, what we obtain is

Lemma 6. The action of $\tilde{G}$ on the set of projectable functions corresponds to

$$
\tilde{G}\left(e_{A}, e_{B}\right)=e_{[A, B]_{+}}-e_{A} \cdot e_{B} .
$$

This implies that for $A=B$ we have

$$
\tilde{G}\left(e_{A}, e_{B}\right)=e_{A^{2}}-e_{A}^{2},
$$

i.e. we find the variance, the quadratic deviation from the mean value.

As a conclusion we get

Corollary 1. $\tilde{G}$ is directly related to the indetermination relations.

\section{EXAmple II: THREE LEVEL QUANTUM SYSTEMS}

6.1. The projective space. In the case of a three level quantum system, the Hilbert space description is done on $\mathbb{C}^{3}$, the operators being (up to multiplication by the imaginary unit) elements of the Lie algebra $\mathfrak{u}(3)$.

The description at the Hilbert space level is fairly similar to the one corresponding to the two levels system. Now the orthonormal basis we consider is $\left\{\left|e_{1}\right\rangle,\left|e_{2}\right\rangle,\left|e_{3}\right\rangle\right\}$ and the corresponding coordinates, complex and real will be $\left\{z_{1}, z_{2}, z_{3}\right\}$ and $\left\{q_{1}, q_{2}, q_{3}, p_{1}, p_{2}, p_{3}\right\}$. The two restrictions:

$$
\langle\psi \mid \psi\rangle=1 \quad|\psi\rangle \in \mathbb{C}^{3}
$$

and

$$
\psi_{1} \sim \psi_{2} \Leftrightarrow \psi_{2}=\lambda \psi_{1} \quad \lambda \in \mathbb{C},
$$

define now the corresponding sphere on the projective space $\mathcal{P} \mathbb{C}$. 
We can read from these relations the definition of the complex and real coordinates and the subsequent expressions for the tensors. But instead of repeating this line of argument, we shall use a different approach and use the momentum mapping $\mu: \mathcal{H} \rightarrow \mathfrak{u}^{*}(3)$ to describe the system.

As we saw above, the relevant structures now shall be those of $\mathfrak{u}^{*}(3)$, which are defined as:

- the Poisson structure corresponds to the canonical Lie-Poisson structure of the dual of a Lie algebra, defined as:

$$
\left\{\alpha_{u}, \alpha_{v}\right\}=\alpha_{[u, v]} \quad \forall u, v \in \mathfrak{u}(3),
$$

where we denote by $\alpha_{u}$ (resp. $\alpha_{v}$ ) the element of $\mathfrak{u}^{*}(3)$ corresponding to the element $u \in \mathfrak{u}(3)$ (resp. $v \in \mathfrak{u}(3)$ ).

- the Riemannian structure is associated to the canonical Jordan structure of the Lie algebra $\mathfrak{u}(3)$. Indeed, we define the tensor $R$

$$
\mathcal{R}(\xi)\left(d \alpha_{u}, d \alpha_{v}\right)=\xi\left([u, v]_{+}\right)=\frac{i}{2} \operatorname{Tr} \xi(u v+v u),
$$

Thus we see that in order to compute the expression of these tensors in some basis, we have to compute the structure constants of the $\mathfrak{u}(3)$ algebra. Thus if we take as basis the one defined by the Gell-Mann matrices

$$
\begin{aligned}
& \lambda_{1}=\left(\begin{array}{lll}
0 & 1 & 0 \\
1 & 0 & 0 \\
0 & 0 & 0
\end{array}\right) \quad \lambda_{2}=\left(\begin{array}{ccc}
0 & -i & 0 \\
i & 0 & 0 \\
0 & 0 & 0
\end{array}\right) \quad \lambda_{3}=\left(\begin{array}{ccc}
1 & 0 & 0 \\
0 & -1 & 0 \\
0 & 0 & 0
\end{array}\right) \\
& \lambda_{4}=\left(\begin{array}{ccc}
0 & 0 & 1 \\
0 & 0 & 0 \\
1 & 0 & 0
\end{array}\right) \quad \lambda_{5}=\left(\begin{array}{ccc}
0 & 0 & -i \\
0 & 0 & 0 \\
i & 0 & 0
\end{array}\right) \quad \lambda_{6}=\left(\begin{array}{ccc}
0 & 0 & 0 \\
0 & 0 & 1 \\
0 & 1 & 0
\end{array}\right) \\
& \lambda_{7}=\left(\begin{array}{ccc}
0 & 0 & 0 \\
0 & 0 & -i \\
0 & i & 0
\end{array}\right) \quad \lambda_{8}=\frac{1}{\sqrt{3}}\left(\begin{array}{ccc}
1 & 0 & 0 \\
0 & 1 & 0 \\
0 & 0 & -2
\end{array}\right) \quad \lambda_{0}=\sqrt{\frac{2}{3}}\left(\begin{array}{ccc}
1 & 0 & 0 \\
0 & 1 & 0 \\
0 & 0 & 1
\end{array}\right)
\end{aligned}
$$

They satisfy the scalar product relations

$$
\operatorname{Tr} \lambda_{\mu} \lambda_{\nu}=2 \delta_{\mu \nu}
$$

Their commutation and anti-commutation relations are written in terms of the antisymmetric structure constants and symmetric d-symbols $d_{\mu \nu \rho}$. We find

$$
\left[\lambda_{\mu}, \lambda_{\nu}\right]=2 i C_{\mu \nu \rho} \lambda_{\rho} \quad\left[\lambda_{\mu}, \lambda_{\rho}\right]_{+}=2 \sqrt{\frac{2}{3}} \lambda_{0} \delta_{\mu \nu}+2 d_{\mu \nu \rho} \lambda_{\rho}
$$

The numerical values turn out to be

$$
C_{123}=1, \quad C_{458}=C_{678}=\frac{\sqrt{3}}{2} C_{147}=-C_{156}=C_{246}=C_{257}=C_{345}=-C_{367}=\frac{1}{2}
$$

The values of these symbols show the different embeddings of $S U(2)$ into $S U(3) \subset$ $U(3)$. For the other coefficients we have 


$$
\begin{gathered}
d_{j j 0}=-d_{0 j j}=-d_{j 0 j}=\sqrt{\frac{2}{3}} \quad j=1, \cdots, 8 \\
-d_{888}=d_{j j 8}=d_{j 8 j}=\frac{1}{\sqrt{3}} \quad j=1,2,3 \\
d_{8 j j}=d_{j j 8}=d_{j 8 j}=-\frac{1}{2 \sqrt{3}} \quad j=4,5,6,7 \\
d_{3 j j}=d_{j j 3}=d_{j 3 j}=\frac{1}{2} \quad j=4,5 \quad d_{3 j j}=d_{j j 3}=d_{j 3 j}=-\frac{1}{2} \quad j=6,7 \\
d_{146}=d_{157}=d_{164}=d_{175}=-d_{247}=d_{256}=d_{265}=-d_{274}=\frac{1}{2} \\
d_{416}=-d_{427}=d_{461}=-d_{472}=d_{517}=d_{526}=d_{562}=d_{571}=\frac{1}{2} \\
d_{614}=d_{625}=d_{641}=d_{652}=d_{715}=-d_{724}=d_{751}=-d_{742}=\frac{1}{2}
\end{gathered}
$$

Thus we have completely characterized the Riemannian and the Poisson tensors along with their associated brackets on the density states of the three-levels system.

\section{EXAmple III: A BIPARTite COMPOSITE SyStem OF TWO SPIN $\frac{1}{2}$ PARTiCles}

Our last example will be devoted to the study of a system composed of two spin$1 / 2$ particles. In this case the Hilbert space is the 4 dimensional complex space $\mathbb{C}^{4}=\mathbb{C}^{2} \otimes \mathbb{C}^{2}$ and the set of Hermitian operators corresponds to the elements of the Lie algebra $\mathfrak{u}(4)$ after multiplication by the imaginary unit.

The importance of the example arises from the fact that this is the simplest situation where entanglement of two systems can be studied.

We will use then the approach used in the previous section, and describe the geometrical structure of the composite system by using the momentum map and thus the image of the Hilbert space $\mathbb{C}^{4}$ on $\mathfrak{u}^{*}(4)$.

7.1. The choice of coordinates on $\mathfrak{u}^{*}(4)$. The most suitable choices corresponds to the tensor product of the basis of the two $\mathfrak{u}(2)$ factors corresponding to each subsystem:

$$
\mathcal{B}=\left\{\sigma_{k} \otimes \tau_{j}\right\}_{k, j=0,1,2,3}
$$

where $\sigma_{0}$ stands for the identity matrix in dimension 2 and $\left\{\sigma_{i}\right\}_{i=1,2,3}$ and $\left\{\tau_{i}\right\}_{i=1,2,3}$ represent two copies of the Pauli matrices. Thus we represent the elements in $\mathfrak{u}(4)$ by a set of 16 real coordinates $\left\{\lambda_{0}, m_{k}, n_{j}, r_{k j}\right\}$ for $k, j=1,2,3$ defined as:

$$
\mathfrak{u}(4) \ni A=\lambda_{0} \sigma_{0} \otimes \tau_{0}+\sum_{k=1}^{3} m_{k} \sigma_{k} \otimes \tau_{0}+\sum_{k=1}^{3} n_{k} \sigma_{0} \otimes \tau_{k}+\sum_{k, j=1}^{3}\left(m_{k} n_{j}+r_{k j}\right) \sigma_{k} \otimes \tau_{j}
$$

Now all tensor fields shall be written in terms of these coordinates in the next sections. This basis has an immediate extension to $\mathfrak{u}^{*}(4)$ by the duality relation defined by the invertibility of the Killing-Cartan form. The main advantage of this choice of coordinates is that separable elements in the space of density operators correspond to the points defined by setting

$$
r_{i j}=0 \quad \forall i, j
$$

7.2. The Riemann and the Poisson structures. The computation of the Poisson and the Riemann tensor requires again to compute the structure constants of the Lie and the Jordan algebras in this basis. Let us present the results by means of the corresponding Hamiltonian and Riemannian vector fields associated to the basis elements: 
7.2.1. Hamiltonian vector fields. Besides the Hamiltonian vector field associated to $\lambda_{0}$, which is a Casimir of the structure, these are the Hamiltonian vector fields associated to the elements of the basis. We list just a few of them, since the others can be obtained from these ones by a simple permutation of indices

$$
\begin{aligned}
& \left\{m_{1}, \cdot\right\}=r_{33} \frac{\partial}{\partial r_{23}}-r_{23} \frac{\partial}{\partial r_{33}}+r_{32} \frac{\partial}{\partial r_{22}}-r_{22} \frac{\partial}{\partial r_{32}}+ \\
& r_{21} \frac{\partial}{\partial r_{31}}-r_{31} \frac{\partial}{\partial r_{21}}-m_{2} \frac{\partial}{\partial m_{3}}+m_{3} \frac{\partial}{\partial m_{2}} \\
& \left\{n_{1}, \cdot\right\}=-r_{32} \frac{\partial}{\partial r_{33}}+r_{33} \frac{\partial}{\partial r_{32}}-r_{22} \frac{\partial}{\partial r_{23}}+r_{23} \frac{\partial}{\partial r_{22}}- \\
& r_{12} \frac{\partial}{\partial r_{13}}+r_{13} \frac{\partial}{\partial r_{12}}-n_{2} \frac{\partial}{\partial n_{3}}+n_{3} \frac{\partial}{\partial n_{2}} \\
& \left\{r_{11}, \cdot\right\}=r_{12} \frac{\partial}{\partial n_{3}}-\left(\left(-1+m_{1}^{2}\right) n_{2}+2 m_{1} r_{12}\right) \frac{\partial}{\partial r_{13}}- \\
& r_{13} \frac{\partial}{\partial n_{2}}+\left(\left(-1+m_{1}^{2}\right) n_{3}+2 m_{1} r_{13}\right) \frac{\partial}{\partial r_{12}}+ \\
& r_{21} \frac{\partial}{\partial m_{3}}-\left(m_{2}\left(-1+n_{1}^{2}\right)+2 n_{1} r_{21}\right) \frac{\partial}{\partial r_{31}}- \\
& r_{31} \frac{\partial}{\partial m_{2}}+\left(m_{3}\left(-1+n_{1}^{2}\right)+2 n_{1} r_{31}\right) \frac{\partial}{\partial r_{21}}+ \\
& \left(m_{2} r_{13}+m_{1}\left(m_{2} n_{3}+r_{23}\right)+n_{2}\left(m_{3} n_{1}+r_{31}\right)+n_{1} r_{32}\right) \frac{\partial}{\partial r_{22}}- \\
& \left(m_{3} r_{12}+n_{3}\left(m_{2} n_{1}+r_{21}\right)+n_{1} r_{23}+m_{1}\left(m_{3} n_{2}+r_{32}\right)\right) \frac{\partial}{\partial r_{33}}+ \\
& \left(-m_{2} r_{12}-m_{1}\left(m_{2} n_{2}+r_{22}\right)+n_{3}\left(m_{3} n_{1}+r_{31}\right)+n_{1} r_{33}\right) \frac{\partial}{\partial r_{23}} \\
& +\left(m_{3} r_{13}-n_{2}\left(m_{2} n_{1}+r_{21}\right)-n_{1} r_{22}+m_{1}\left(m_{3} n_{3}+r_{33}\right)\right) \frac{\partial}{\partial r_{32}} \\
& \left\{r_{12}, \cdot\right\}=r_{11} \frac{\partial}{\partial n_{3}}-\left(\left(-1+m_{1}^{2}\right) n_{1}+2 m_{1} r_{11}\right) \frac{\partial}{\partial r_{13}}- \\
& r_{13} \frac{\partial}{\partial n_{1}}+\left(\left(-1+m_{1}^{2}\right) n_{3}+2 m_{1} r_{13}\right) \frac{\partial}{\partial r_{11}} \\
& -r_{22} \frac{\partial}{\partial m_{3}}+\left(m_{2}\left(-1+n_{2}^{2}\right)+2 n_{2} r_{22}\right) \frac{\partial}{\partial r_{32}}+ \\
& \left(-m_{3} r_{11}+n_{3}\left(m_{2} n_{2}+r_{22}\right)+n_{2} r_{23}-m_{1}\left(m_{3} n_{1}+r_{31}\right)\right) \frac{\partial}{\partial r_{33}}+r_{32} \frac{\partial}{\partial m_{2}}+ \\
& \left(m_{2} r_{13}+m_{1}\left(m_{2} n_{3}+r_{23}\right)-n_{2}\left(m_{3} n_{1}+r_{31}\right)-n_{1} r_{32}\right) \frac{\partial}{\partial r_{21}}- \\
& \left(m_{3}\left(-1+n_{2}^{2}\right)+2 n_{2} r_{32}\right) \frac{\partial}{\partial r_{22}}- \\
& \left(m_{2} r_{11}+m_{1}\left(m_{2} n_{1}+r_{21}\right)+n_{3}\left(m_{3} n_{2}+r_{32}\right)+n_{2} r_{33}\right) \frac{\partial}{\partial r_{23}}+ \\
& \left(m_{3} r_{13}+n_{2}\left(m_{2} n_{1}+r_{21}\right)+n_{1} r_{22}+m_{1}\left(m_{3} n_{3}+r_{33}\right)\right) \frac{\partial}{\partial r_{31}}
\end{aligned}
$$


BASICS OF QUANTUM MECHANICS, GEOMETRIZATION AND SOME APPLICATIONS .. 29

$$
\begin{aligned}
\left\{r_{21}, \cdot\right\}= & r_{11} \frac{\partial}{\partial m_{3}}-\left(m_{1}\left(-1+n_{1}^{2}\right)+2 n_{1} r_{11}\right) \frac{\partial}{\partial r_{31}}- \\
& r_{22} \frac{\partial}{\partial n_{3}}+\left(\left(-1+m_{2}^{2}\right) n_{2}+2 m_{2} r_{22}\right) \frac{\partial}{\partial r_{23}}+ \\
& r_{23} \frac{\partial}{\partial n_{2}}-\left(\left(-1+m_{2}^{2}\right) n_{3}+2 m_{2} r_{23}\right) \frac{\partial}{\partial r_{22}}- \\
& r_{31} \frac{\partial}{\partial m_{1}}+\left(m_{3}\left(-1+n_{1}^{2}\right)+2 n_{1} r_{31}\right) \frac{\partial}{\partial r_{11}}+ \\
& \left(-m_{2} r_{13}-m_{1}\left(m_{2} n_{3}+r_{23}\right)+n_{2}\left(m_{3} n_{1}+r_{31}\right)+n_{1} r_{32}\right) \frac{\partial}{\partial r_{12}}+ \\
& \left(-n_{3}\left(m_{1} n_{1}+r_{11}\right)-n_{1} r_{13}+m_{3} r_{22}+m_{2}\left(m_{3} n_{2}+r_{32}\right)\right) \frac{\partial}{\partial r_{33}}+ \\
& \left(m_{2} r_{12}+m_{1}\left(m_{2} n_{2}+r_{22}\right)+n_{3}\left(m_{3} n_{1}+r_{31}\right)+n_{1} r_{33}\right) \frac{\partial}{\partial r_{13}}- \\
& \left(n_{2}\left(m_{1} n_{1}+r_{11}\right)+n_{1} r_{12}+m_{3} r_{23}+m_{2}\left(m_{3} n_{3}+r_{33}\right)\right) \frac{\partial}{\partial r_{32}}
\end{aligned}
$$

7.2.2. Riemannian vector fields. Also by using the Riemannian structure we can associate vector fields with coordinate functions. Again, we just list a few of them:

$$
\begin{aligned}
J_{\lambda_{0}}= & n_{1} \frac{\partial}{\partial n_{1}}+n_{2} \frac{\partial}{\partial n_{2}}+n_{3} \frac{\partial}{\partial n_{3}}+m_{1} \frac{\partial}{\partial m_{1}}+m_{2} \frac{\partial}{\partial m_{2}}+m_{3} \frac{\partial}{\partial m_{3}}+y_{0} \frac{\partial}{\partial y_{0}}+ \\
& r_{11} \frac{\partial}{\partial r_{11}}+r_{12} \frac{\partial}{\partial r_{12}}+r_{13} \frac{\partial}{\partial r_{13}}+r_{21} \frac{\partial}{\partial r_{21}}+r_{22} \frac{\partial}{\partial r_{22}}+r_{23} \frac{\partial}{\partial r_{23}}+ \\
& r_{31} \frac{\partial}{\partial r_{31}}+r_{32} \frac{\partial}{\partial r_{32}}+r_{33} \frac{\partial}{\partial r_{33}}-n_{1} m_{1} \frac{\partial}{\partial r_{11}}-n_{2} m_{1} \frac{\partial}{\partial r_{12}}-n_{3} m_{1} \frac{\partial}{\partial r_{13}}+ \\
& -n_{1} m_{2} \frac{\partial}{\partial r_{21}}-n_{2} m_{2} \frac{\partial}{\partial r_{22}}-n_{3} m_{2} \frac{\partial}{\partial r_{23}}+-n_{1} m_{3} \frac{\partial}{\partial r_{31}}-n_{2} m_{3} \frac{\partial}{\partial r_{32}}-n_{3} m_{3} \frac{\partial}{\partial r_{33}} \\
J_{m_{1}}= & y_{0} \frac{\partial}{\partial m_{1}}+\left(m_{1} n_{1}+r_{11}\right) \frac{\partial}{\partial n_{1}}+\left(m_{1} n_{2}+r_{12}\right) \frac{\partial}{\partial n_{2}}+\left(m_{1} n_{3}+r_{13}\right) \frac{\partial}{\partial n_{3}}- \\
& \left(m_{1} r_{11}+n_{1}\left(m_{1}^{2}+y_{0}-1\right)\right) \frac{\partial}{\partial r_{11}}-\left(m_{1} r_{12}+n_{2}\left(m_{1}^{2}+y_{0}-1\right)\right) \frac{\partial}{\partial r_{12}}- \\
& \left(m_{1} r_{13}+n_{3}\left(m_{1}^{2}+y_{0}-1\right)\right) \frac{\partial}{\partial r_{13}}- \\
& m_{2}\left(m_{1} n_{1}+r_{11}\right) \frac{\partial}{\partial r_{21}}-m_{2}\left(m_{1} n_{2}+r_{12}\right) \frac{\partial}{\partial r_{22}}- \\
& m_{2}\left(m_{1} n_{3}+r_{13}\right) \frac{\partial}{\partial r_{23}}-m_{3}\left(m_{1} n_{1}+r_{11}\right) \frac{\partial}{\partial r_{31}}- \\
& m_{3}\left(m_{1} n_{2}+r_{12}\right) \frac{\partial}{\partial r_{32}}-m_{3}\left(m_{1} n_{3}+r_{13}\right) \frac{\partial}{\partial r_{33}}+ \\
& m_{1} \frac{\partial}{\partial y_{0}}
\end{aligned}
$$




$$
\begin{aligned}
J_{n_{1}}= & \left(m_{1} n_{1}+r_{11}\right) \frac{\partial}{\partial m_{1}}+\left(m_{2} n_{1}+r_{21}\right) \frac{\partial}{\partial m_{2}}+ \\
& \left(m_{3} n_{1}+r_{31}\right) \frac{\partial}{\partial m_{3}}+y_{0} \frac{\partial}{\partial n_{1}}-\left(n_{1} r_{11}+m_{1}\left(n_{1}^{2}+y_{0}-1\right)\right) \frac{\partial}{\partial r_{11}}- \\
& n_{2}\left(m_{1} n_{1}+r_{11}\right) \frac{\partial}{\partial r_{12}}-n_{3}\left(m_{1} n_{1}+r_{11}\right) \frac{\partial}{\partial r_{13}}- \\
& \left(n_{1} r_{21}+m_{2}\left(n_{1}^{2}+y_{0}-1\right)\right) \frac{\partial}{\partial r_{21}}-n_{2}\left(m_{2} n_{1}+r_{21}\right) \frac{\partial}{\partial r_{22}}-n_{3}\left(m_{2} n_{1}+r_{21}\right) \frac{\partial}{\partial r_{23}}- \\
& \left(n_{1} r_{31}+m_{3}\left(n_{1}^{2}+y_{0}-1\right)\right) \frac{\partial}{\partial r_{31}}-n_{2}\left(m_{3} n_{1}+r_{31}\right) \frac{\partial}{\partial r_{32}}-n_{3}\left(m_{3} n_{1}+r_{31}\right) \frac{\partial}{\partial r_{33}}+n_{1} \frac{\partial}{\partial y_{0}} \\
J_{r_{11}}= & \left(m_{1} r_{11}+n_{1}\left(m_{1}^{2}+y_{0}-1\right)\right) \frac{\partial}{\partial m_{1}}+m_{1}\left(m_{2} n_{1}+r_{21}\right) \frac{\partial}{\partial m_{2}}+ \\
& m_{1}\left(m_{3} n_{1}+r_{31}\right) \frac{\partial}{\partial m_{3}}+\left(n_{1} r_{11}+m_{1}\left(n_{1}^{2}+y_{0}-1\right)\right) \frac{\partial}{\partial n_{1}}+ \\
& n_{1}\left(m_{1} n_{2}+r_{12}\right) \frac{\partial}{\partial n_{2}}+n_{1}\left(m_{1} n_{3}+r_{13}\right) \frac{\partial}{\partial n_{3}}- \\
& \left(\left(2 n_{1}^{2}+y_{0}-2\right) m_{1}^{2}+2 n_{1} r_{11} m_{1}+n_{1}^{2}\left(y_{0}-2\right)+y_{0}\right) \frac{\partial}{\partial r_{11}}- \\
& \left(m_{1} n_{2} r_{11}+n_{1}\left(m_{1} r_{12}+n_{2}\left(2 m_{1}^{2}+y_{0}-2\right)\right)\right) \frac{\partial}{\partial r_{12}}- \\
& \left(m_{1} n_{3} r_{11}+n_{1}\left(m_{1} r_{13}+n_{3}\left(2 m_{1}^{2}+y_{0}-2\right)\right)\right) \frac{\partial}{\partial r_{13}}- \\
& \left(m_{2} n_{1} r_{11}+m_{1}\left(n_{1} r_{21}+m_{2}\left(2 n_{1}^{2}+y_{0}-2\right)\right)\right) \frac{\partial}{\partial r_{21}}+ \\
& \left(m_{3} n_{3}-m_{2} n_{1} r_{12}-m_{1} n_{2}\left(2 m_{2} n_{1}+r_{21}\right)+r_{33}\right) \frac{\partial}{\partial r_{22}}- \\
& \left(m_{3} n_{2}+m_{2} n_{1} r_{13}+m_{1} n_{3}\left(2 m_{2} n_{1}+r_{21}\right)+r_{32}\right) \frac{\partial}{\partial r_{23}}- \\
& \left(m_{3} n_{1} r_{11}+m_{1}\left(n_{1} r_{31}+m_{3}\left(2 n_{1}^{2}+y_{0}-2\right)\right)\right) \frac{\partial}{\partial r_{31}}- \\
& \left(m_{2} n_{3}+m_{3} n_{1} r_{12}+r_{23}+m_{1} n_{2}\left(2 m_{3} n_{1}+r_{31}\right)\right) \frac{\partial}{\partial r_{32}}+ \\
& \left(m_{2} n_{2}-m_{3} n_{1} r_{13}+r_{22}-m_{1} n_{3}\left(2 m_{3} n_{1}+r_{31}\right)\right) \frac{\partial}{\partial r_{33}}+\left(m_{1} n_{1}-r_{11}\right) \frac{\partial}{\partial y_{0}} \\
&
\end{aligned}
$$




$$
\begin{aligned}
J_{r_{12}}= & \left(m_{1} r_{12}+n_{2}\left(m_{1}^{2}+y_{0}-1\right)\right) \frac{\partial}{\partial m_{1}}+m_{1}\left(m_{2} n_{2}+r_{22}\right) \frac{\partial}{\partial m_{2}}+ \\
& m_{1}\left(m_{3} n_{2}+r_{32}\right) \frac{\partial}{\partial m_{3}}+n_{2}\left(m_{1} n_{1}+r_{11}\right) \frac{\partial}{\partial n_{1}}+ \\
& \left(n_{2} r_{12}+m_{1}\left(n_{2}^{2}+y_{0}-1\right)\right) \frac{\partial}{\partial n_{2}}+n_{2}\left(m_{1} n_{3}+r_{13}\right) \frac{\partial}{\partial n_{3}}- \\
& \left(m_{1} n_{2} r_{11}+n_{1}\left(m_{1} r_{12}+n_{2}\left(2 m_{1}^{2}+y_{0}-2\right)\right)\right) \frac{\partial}{\partial r_{11}}- \\
& \left(\left(2 n_{2}^{2}+y_{0}-2\right) m_{1}^{2}+2 n_{2} r_{12} m_{1}+n_{2}^{2}\left(y_{0}-2\right)+y_{0}\right) \frac{\partial}{\partial r_{12}}- \\
& \left(m_{1} n_{3} r_{12}+n_{2}\left(m_{1} r_{13}+n_{3}\left(2 m_{1}^{2}+y_{0}-2\right)\right)\right) \frac{\partial}{\partial r_{13}}- \\
& \left(m_{3} n_{3}+m_{2} n_{2} r_{11}+m_{1} n_{1}\left(2 m_{2} n_{2}+r_{22}\right)+r_{33}\right) \frac{\partial}{\partial r_{21}}- \\
& \left(m_{2} n_{2} r_{12}+m_{1}\left(n_{2} r_{22}+m_{2}\left(2 n_{2}^{2}+y_{0}-2\right)\right)\right) \frac{\partial}{\partial r_{22}}+ \\
& \left(m_{3} n_{1}-m_{2} n_{2} r_{13}-m_{1} n_{3}\left(2 m_{2} n_{2}+r_{22}\right)+r_{31}\right) \frac{\partial}{\partial r_{23}}+ \\
& \left(m_{2} n_{3}-m_{3} n_{2} r_{11}+r_{23}-m_{1} n_{1}\left(2 m_{3} n_{2}+r_{32}\right)\right) \frac{\partial}{\partial r_{31}}- \\
& \left(m_{3} n_{2} r_{12}+m_{1}\left(n_{2} r_{32}+m_{3}\left(2 n_{2}^{2}+y_{0}-2\right)\right)\right) \frac{\partial}{\partial r_{32}}- \\
& \left(m_{2} n_{1}+m_{3} n_{2} r_{13}+r_{21}+m_{1} n_{3}\left(2 m_{3} n_{2}+r_{32}\right)\right) \frac{\partial}{\partial r_{33}}+\left(m_{1} n_{2}-r_{12}\right) \frac{\partial}{\partial y_{0}}
\end{aligned}
$$

By direct computation, but also form general considerations, these vector fields generate the Lie algebra of $G L(3, \mathbb{C})$. This algebra contains Hamiltonian vector fields and vector fields which describe dissipation or decoherence. These results are related to those of Rajeev (31]).

7.3. The analysis of entanglement. Entanglement is a property of composite physical systems which plays a very important role in many different phenomena, but in particular, it has become a crucial issue of quantum computation and quantum information theory. Despite the growing interest in recent years, it was already discussed by Schrödinger and the "founding fathers" of quantum theory in the early years of quantum theory ([32, 33] ).

Roughly speaking, entanglement is the situation complementary to separability

Definition 3. Let $|\psi\rangle$ be state of a Hilbert space $\mathcal{H}=\mathcal{H}_{1} \otimes \mathcal{H}_{2}$ of a bipartite system. Then, $|\psi\rangle$ is said to be separable if there exists a pair of states $\left|\psi_{1}\right\rangle \in \mathcal{H}_{1}$ and $\left|\psi_{2}\right\rangle \in \mathcal{H}_{2}$ satisfying $|\psi\rangle=\left|\psi_{1}\right\rangle \otimes\left|\psi_{2}\right\rangle$.

Thus a state which is not separable is said to be entangled. But entanglement exhibits many interesting properties, for instance the fact that there is a gradation in the level of entanglement of the different states. Thus we can measure the entanglement of a state by using physical quantities. These different observables are called entanglement witnesses.

Usual choices to describe entanglement are the concurrence of the state, the von Neumann entropy of one of its partial traces (i.e. the entropy of the density state $\rho_{1}=\operatorname{Tr}_{2} \rho_{\psi}$ or of $\rho_{2}=\operatorname{Tr}_{1} \rho_{\psi}$, where $\left.\rho_{\psi}=|\psi\rangle\langle\psi|\right)$.

Definition 4. The concurrence of a density matrix $\rho \in \mathcal{D}(\mathcal{H})$ is defined as

$$
C(\rho)=\max \left(0,2 \lambda_{\max }(\hat{\rho})-\operatorname{Tr}(\hat{\rho})\right)
$$

where $\hat{\rho}$ corresponds to

$$
\hat{\rho}=\sqrt{\left(\sigma_{2} \otimes \sigma_{2}\right) \rho^{*}\left(\sigma_{2} \otimes \sigma_{2}\right) \rho}
$$


and $\lambda_{\max }(\hat{\rho})$ stands for its largest eigenvalue.

Definition 5. The von Neumann entropy of a density matrix $\rho \in \mathcal{D}(\mathcal{H})$ is defined as

$$
S(\rho)=\operatorname{Tr} \rho \log (\rho) .
$$

When the density matrix corresponds to a pure state, though, the function above vanishes. Thus we define the corresponding entropy as the value of the function on the partial trace over one of the subsystems:

$$
S\left(\rho_{\psi}\right)=\operatorname{Tr} \rho_{1} \log \left(\rho_{1}\right) \quad \rho_{1}=\operatorname{Tr}_{1} \rho_{\psi}
$$

On the other hand, if we consider the case of mixed states, the situation is not equally simple. In general, it is necessary to consider more than one entanglement witnesses in order to completely characterize the state of the system. One interesting question arises thus: how can we describe the independence of the different quantities we use?

In the framework of classical mechanics this question is simple to answer. Given two physical quantities, which are represented by two functions $f_{1}, f_{2}$ on phase-space, they are said to be independent at a point $p$ if their exterior differentials satisfy

$$
\left(d f_{1} \wedge d f_{2}\right)(p) \neq 0
$$

The usual approach to Quantum Mechanics, in terms of Hilbert spaces or $\mathbb{C}^{*}$-algebras does not allow a similar treatment of the analogous quantum problem. We lack of a well developed non-commutative differential calculus which would allow to define a "noncommutative" exterior differential and therefore to extend the previous definition to the quantum setting [27, 34, 35, 4]

However the geometrical formalism we introduced in the previous sections allows us to look at the problem from a different perspective. Treating the quantum state space as a real differential manifold, we do have a differential calculus at our disposal: the usual differential calculus of real manifolds.

Consider the Hilbert space $\mathcal{H}$ and an operator $A$. We know that we can associate with $A$ the quadratic function

$$
A \rightarrow f_{A}(\psi)=\langle\psi|A| \psi\rangle \quad \psi \in \mathcal{H} .
$$

In the geometric description of Quantum Mechanics we read from the set of quadratic functions the algebraic structures available on the set of operators:

- the associative product of operators is translated into the non-local product $\star$,

- the Lie algebra defined by the commutator is translated into the Poisson algebra defined by the tensor $\Lambda$

- the Jordan algebra given by the anticommutator is translated into the Jordan algebra defined by the tensor $G$

But the geometric description also includes a pointwise algebra $\left(f_{A} \cdot f_{B}\right)(\psi)=f_{A}(\psi) f_{B}(\psi)$, which is commutative, and whose differential calculus is the standard one. This is the algebraic structure with respect to which we define the differential algebra we are interested in:

Definition 6. Two observables $A$ and $B$ are said to be functionally independent if their associated functions satisfy

$$
d f_{A} \wedge d f_{B} \neq 0 \text { on a dense submanifold of } \mathcal{H}
$$

This is a condition which is simple to test and exhibits a clear advantage offered by the geometric approach to the description of quantum systems, for there is no simple analogue of this structure in the usual framework.

Let us see how this works in a particular example where we can test the independence of von Neumann entropy and the concurrence of our two qubit system: 
Example 1. Now we will test this formalism with a particular example. Consider for instance the family of density states defined by the matrices:

$$
\rho_{t}=\left(\begin{array}{cccc}
0 & 0 & 0 & 0 \\
0 & a & \frac{1}{2} c e^{i \phi}, 0 & \\
0 & \frac{1}{2} c e^{-i \phi} & b & 0 \\
0 & 0 & 0 & 1-a-b
\end{array}\right)
$$

as it is considered in [12]. Such a matrix represents a density state provided that

$$
0 \leq a+b \leq 1 \quad 0 \leq c \leq 1 \quad 4 a b \geq c
$$

This is clearly a 4-dimensional submanifold $\mathcal{S}$ of $\mathcal{D}(\mathcal{H})$. We can take an adapted basis for it, considering the matrices

$$
\left(\begin{array}{cccc}
0 & 0 & 0 & 0 \\
0 & 1 & 0 & 0 \\
0 & 0 & 0 & 0 \\
0 & 0 & 0 & -1
\end{array}\right),\left(\begin{array}{cccc}
0 & 0 & 0 & 0 \\
0 & 0 & 0 & 0 \\
0 & 0 & 1 & 0 \\
0 & 0 & 0 & -1
\end{array}\right),\left(\begin{array}{cccc}
0 & 0 & 0 & 0 \\
0 & 0 & 1 & 0 \\
0 & 1 & 0 & 0 \\
0 & 0 & 0 & 0
\end{array}\right),\left(\begin{array}{cccc}
0 & 0 & 0 & 0 \\
0 & 0 & i & 0 \\
0 & -i & 0 & 0 \\
0 & 0 & 0 & 0
\end{array}\right)
$$

We can use the four real numbers $\{a, b, c, \phi\}$ as adapted coordinates on that submanifold.

Now we can evaluate the three functions above on these states. As we already know the expression of the Jordan and the Poisson bracket we can also obtain the corresponding Hamiltonian and gradient vector fields. And besides, we can also study the independence of the functions by evaluating the expressions of

$$
d S \wedge d C
$$

where $d$ represents the exterior differential associated with the differential manifold constituted by $\mathfrak{u}^{*}(4)$.

Let us thus proceed:

- The value of the different functions is easy to obtain. We have that von Neumann entropy reads,

$$
\begin{aligned}
2 S\left(\rho_{t}\right)= & -2(-1+a+b) \log [1-a-b]+ \\
& \left(a+b-\sqrt{(a-b)^{2}+c^{2}}\right) \log \left[\frac{1}{2}\left(a+b-\sqrt{(a-b)^{2}+c^{2}}\right)\right]+ \\
& \left(a+b+\sqrt{(a-b)^{2}+c^{2}}\right) \log \left[\frac{1}{2}\left(a+b+\sqrt{(a-b)^{2}+c^{2}}\right)\right] .
\end{aligned}
$$

The value of the concurrence is very simple:

$$
C\left(\rho_{t}\right)=c
$$

- We can study now the Poisson brackets corresponding to them. It is simple to prove that these functions commute, i.e.

$$
\{S, C\}=0
$$

This implies that the local transformations generated by them commute.

- Finally, we can study the independence of the different functions. This is an important issue, in particular the independence of the von Neumann entropy and the concurrence, because it affects the description of entanglement of general quantum density states. We can prove the following:

Lemma 7. The concurrence and the von Neumann entropy of the family of states $\rho_{t}$ are not independent in all the space of density states.

Proof. We are considering the submanifold of $\mathfrak{u}(\mathcal{H})$ corresponding to the family of density states $\rho_{t}$.

Thus the differential of the concurrence is trivial to obtain:

$$
d C\left(\rho_{t}\right)=d c .
$$


The computation of the differential of the von Neumann entropy is quite more involved. It is evident from the expression above that the functions $\mathcal{S}$ depends on the three variables. But as $C$ depends only on $c$, we have to consider only the $a$ and $b$ dependence in what regards the computation of $d S \wedge d C$. To this aim we compute:

$$
\frac{\partial S}{\partial a}, \quad \frac{\partial S}{\partial b}
$$

Now, the condition for 50 to be equal to zero corresponds to

And these conditions become

$$
\frac{\partial S}{\partial a}=0=\frac{\partial S}{\partial b}
$$

$2 \log [1-a-b]+\log \left[a b-\frac{c^{2}}{4}\right]=0 \quad \log \left[4 a b-c^{2}\right]-2 \log \left[a+b+\sqrt{(a-b)^{2}+c^{2}}\right]=0$

These equations have a solution on

$$
\frac{1}{3}<a<\frac{1}{2} ; \quad b=a ; \quad c=\sqrt{-4+8 a-4 a^{2}+8 b-4 a b-4 b^{2}}
$$

If we represent the condition for $c$ as a function of $a$ and $b$ we verify that it is well defined for all values of $a$ and $b$ (we represent the function $c=\sqrt{-4+8 a-4 a^{2}+8 b-4 a b-4 b^{2}}$ and the function $c=\sqrt{-a+16 a-12 a^{2}}$, which corresponds to the evaluation on the submanifold $a=b)$.

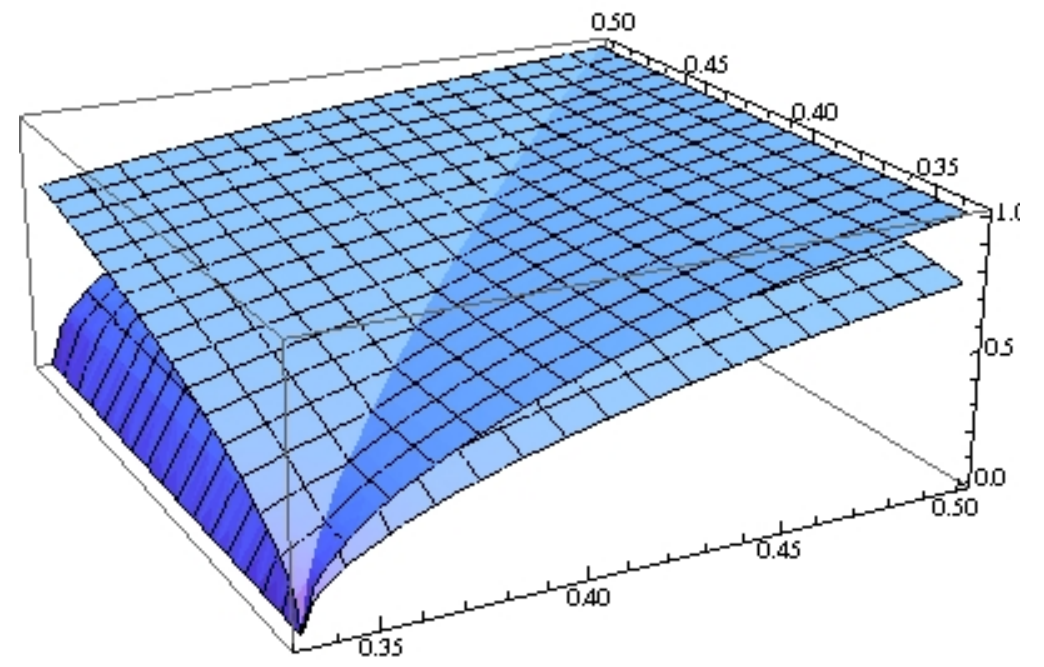

Thus we conclude that there is a nonempty subset of $\mathfrak{u}^{*}(4)$ where the von Neumann entropy functions and the concurrence function introduced above are not functionally independent. On any point outside this submanifold the two functions are indeed functionally independent.

\section{Conclusions And outlook}

To summarize the exposition we would like to mention a few advantages and some potential applications of the geometric approach to Quantum Mechanics that we just presented to the field of Quantum Information Theory and Quantum Control. 
The main idea the paper aims to transmit is that Quantum Mechanics can be efficiently presented as a geometric theory, considering that a quantum dynamical system can be defined as

- a dynamical system on a Kähler manifold where the dynamical vector field is Hamiltonian with respect to the canonical symplectic structure and is a Killing vector for the Riemannian metric.

- a dynamical system defined as a derivation for an associative algebra endowed with a pair of operations which define a Lie-Jordan algebra on it.

In both cases, the message is simple: the geometric framework provides an approach to quantum mechanics which is very close to the usual geometric framework of classical mechanics and many of the "classical tools" can therefore be used in the quantum domain. The most relevant is perhaps the differential structure encoded in the exterior differential calculus. Such a structure has not a simple analogue in the usual quantum description, but provides a powerful tool to study important notions as the independence of operators, as we saw in the last section. Also the passage to the projective space turns out to be a simple task for, up to some conformal factors whose meaning is clear in geometrical terms, the treatment of the projective theory is analogous to the one defined at the level of Hilbert spaces.

But the analogies of the quantum and the classical frameworks have also some other deeper implications, particularly in the realm of Quantum Computation and Quantum Information theory. We have seen how the cases of one and two qubits and one qutrit can be perfectly described within the geometric approach in fairly simple terms. The geometric implications of entanglement is under study now, and we hope to be able to provide a detailed description in a short time. But also quantum channels, their capacities, the study of decoherence, etc are concepts with a simple translation in the geometric domain. We are presently addressing these issues and they will appear elsewhere.

Finally we would like to mention another advantage of the geometric description of quantum mechanics: the study of quantum control problems. Geometric control theory is a well stablished theory which has provided very powerful tools to describe classical control systems. On the other hand, the usual description of quantum control problems makes difficult to translate or adapt those classical tools to the case of quantum systems. But once we have proved that quantum dynamical systems can be described using the same mathematics which is used in the classical case, the possibilities for application of classical geometric control theory seem to be quite rich. And this is even true for systems and phenomena purely quantum in origin, as the quantum Zeno effect, whose applications to control problems are becoming an interesting topic [38, 19, 18.

\section{REFERENCES}

[1] P. Aniello, V. I. Man'ko, G. Marmo, S. Solimeno, and F. Zaccaria. On the coherent states, dislacement operators and quasidistributions associated with deformed oscillators. J. Opt. B Quantum Semiclass. Opt, 2(6):718-725, 2000.

[2] Carl M. Bender. Making sense of non-hermitian hamiltonians. Rep. Prog. Phys., 70:947-1018, 2007.

[3] K. Berndl, D. Dürr, S. Goldstein, G. Peruzzi, and N. Zanghi. On the global existence of bohmian mechanics. Comm. Math. Phys., 173:647-673, 1995.

[4] G. Bimonte, G. Marmo, and A. Stern. Bicovariant calculus in quantum theory and a generalization of the gauss law. Phys Lett B, 478:358, 2000.

[5] J. F. Cariñena, J. Clemente-Gallardo, and G. Marmo. Geometrization of Quantum Mechanics. Theor. Math Phys, 152(1):894-903, 2007.

[6] J. F. Cariñena, J. Clemente-Gallardo, and G. Marmo. Proceedings of the XV International Workshop on Geometry and Physics, volume 11 of Publicaciones de la RSME, chapter Introduction to Quantum Mechanics and the Quantum-Classical transition, pages 3-45. RSME, 2007. 
[7] D. Chruscinski and G. Marmo. Remarks on the geometrical description of the GNS construction. Technical report, Univ. Naples, 2008.

[8] R. Cirelli, A. Maniá, and L. Pizzocchero. Quantum Mechanics as an infinite-dimensional Hamiltonian system with uncertainty structure i. J. Math Phys, 31(12):2891-2897, 1990.

[9] R. Cirelli, A. Maniá, and L. Pizzocchero. Quantum phase-space formulation of Schrödinger mechanics. J. Mod Phys A, 6(12):2133-2146, 1991.

[10] R. Cirelli, A. Maniá, and L. Pizzocchero. A functional representation for noncommutative $\mathbb{C}^{*}$-algebras. Rev Math Phys, 6(5):675-697, 1994.

[11] J. Clemente-Gallardo and G. Marmo. Differential Geometric Methods in Mechanics and Field Theory, chapter The space of density states in geometrical quantum mechanics, pages 35-56. Gent Academia Press, 2007.

[12] L. Derkacz and L. Jakobczyk. CHSH violation and entropy - concurrence plane. Phys. Rev. A, 72,:042321, 2005.

[13] P. A. M. Dirac. The principles of Quantum Mechanics. Clarendon Press, Oxford, 2 edition, 1936.

[14] O. Donati, G. F. Missiroli, and G. Pozzi. An experiment on electron interference. Am. J. Phys, 41:639, 1973

[15] B. Dubrovin, M. Giordano, G. Marmo, and A. Simoni. Poisson brackets on presymplectic manifolds. Int. J. Mod. Phys. A, 8(21):3747-3771, 1993

[16] G. G. Emch. Foundations of 20th century Physics. North Holland, 1984.

[17] G. Esposito, G. Marmo, and G. Sudarshan. From Classical to Quantum Mechanics: an introduction to the formalism. Cambridge Univ Press, 2004.

[18] P. Facchi, G. Marmo, and S. Pascazio. Quantum Zeno dynamics and quantum Zeno subspaces. Technical report, arxiv:0711:4280, 2007.

[19] P. Facchi, S. Tasaki, S. Pascazio, H. Nakazato, A. Tokuse, and D. A. Lidar. Control of decoherence: Analysis and comparison of three different strategies. Phys Rev A, 71:022302, 2005.

[20] Janusz Grabowski, Marek Kus, and Giuseppe Marmo. Geometry of quantum systems: density states and entanglement. J.Phys. A, 38:10217-10244, 2005.

[21] Janusz Grabowski, Marek Kus, and Giuseppe Marmo. Symmetries, group actions, and entanglement. Open Syst. Inf. Dyn., 13:343-362, 2006.

[22] N. P. Landsman. Mathematical topics between Classical and Quantum Mechanics. SpringerVerlag, 1998.

[23] O. V. Man'ko, V. I. Man'ko, and G. Marmo. Alternative commutation relations, star products and tomography. J. Phys. A, 35:699-719, 2002.

[24] V. I. Man'ko, G. Marmo, A. Simoni, and F. Ventriglia. Tomography in abstract hilbert spaces. Open Systems \& Information Dynamics, 13(3):239-253, 2006.

[25] V. I. Manko, G. Marmo, E.C.G. Sudarshan, and F. Zaccaria. Differential geometry of density states. Rep. Math. Phys, 55:405-422, 2005.

[26] G. Marmo, E. J. Saletan, A. Simoni, and B. Vitale. Dynamical Systems, a differential geometric approach to symmetry and reduction. John Wiley, Chicester, 1985.

[27] G. Marmo, P. Vitale, and A. Zampini. Noncommutative differential calculus for Moyal subalgebras. J. Geom. Phys., 55:611-622, 2006.

[28] P. G. Merli, G. F. Missiroli, and G. Pozzi. On the statistical aspects of electron interference phenomena. Am. J. Phys, 44:306, 1976.

[29] A. Mostafazadeh. Pseudo-hermiticity versus pt symmetry: the necessary conditions for the reality of the spectrum of a nonhermitian Hamiltonian. J. Math. Phys., 43:2814, 2002.

[30] J. E. Moyal. Quantum mechanics as a statistical theory. Proc. Cambridge Phil. Soc., 45:99$124,1949$.

[31] S.G. Rajeev. A canonical formulation of dissipative mechanics using complex-valued Hamiltonians. Annals of Physics, 322(7):1541-1555, 2007.

[32] E. Schrödinger. Discussion of probability relations between separated systems. Proceedings of the Cambridge Philosophical Society, 31:555-563, 1935.

[33] E. Schrödinger. Discussion of probability relations between separated systems. Proceedings of the Cambridge Philosophical Society, 32:446-451, 1936.

[34] I. E. Segal. Quantized differential forms. Topology, 8:147, 1967.

[35] I. E. Segal. Quantization of the de rham complex. Proc. Symp. Pure Math, (16):205, 1970. 
BASICS OF QUANTUM MECHANICS, GEOMETRIZATION AND SOME APPLICATIONS .. 37

[36] P. Sonnentag. Ein Experiment zur kontrollierten Dekohärenz in einem ElektronenBiprismaInterferometer. $\mathrm{PhD}$ thesis, University of Tubingen, 2006.

[37] J. Steeds, P. G. Merli, G. Pozzi, G. Missiroli, and A. Tonomura. The double-slit experiment with single electrons. Phys. World, 16:20, 2003.

[38] S. Tasaki, A. Tokuse, P. Facchi, and S. Pascazio. Control of decoherence: Dynamical decoupling versus quantum Zeno effect: A case study for trapped ions. International Journal of Quantum Chemistry, 198(2):160-172, 2004.

[39] A. Tonomura, J. Endo, T. Matsuda, T. Kawasaki, and H. Ezawa. Demonstration of singleelectron build up of an interference pattern. Am. J. Phys, 57:117-120, 1989.

[40] V.S. Varadarajan. Geometry of quantum theory I and II. Indian Statistical Inst., 1965.

[41] F. Ventriglia. Alterntive Hamiltonian descriptions for quantum systems and non-hermitian operators with real spectrum. Mod Phys Lett A, 17:1589, 2002.

[42] E. P. Wigner. Quantum corrections for thermodynamic equilibrium. Phys. Rev, 40:749-759, 1932 .

Instituto de Biocomputación y Física de los Sistemas Complejos, Universidad de Zaragoza, Corona de Aragón 42, 50009 Zaragoza (SPAIN)

Dipartamento di Scienze Fisiche, Universitá Federico it and inFN sezione di Napoli, Via Cintia, 80126 NAPOLI (ITALY) 